\title{
SPHERICAL SUBMANIFOLDS WITH FINITE TYPE SPHERICAL GAUSS MAP
}

\author{
Bang-Yen Chen and Huei-Shyong Lue
}

\begin{abstract}
The study of Euclidean submanifolds with finite type "classical" Gauss map was initiated by B.-Y. Chen and P. Piccinni in [11]. On the other hand, it was believed that for spherical submanifolds the concept of spherical Gauss map is more relevant than the classical one (see [20]). Thus the purpose of this article is to initiate the study of spherical submanifolds with finite type spherical Gauss map. We obtain several fundamental results in this respect. In particular, spherical submanifolds with 1-type spherical Gauss map are classified. From which we conclude that all isoparametric hypersurfaces of $S^{n+1}$ have 1-type spherical Gauss map. Among others, we also prove that Veronese surface and equilateral minimal torus are the only minimal spherical surfaces with 2-type spherical Gauss map.
\end{abstract}

\section{Introduction}

Let $M^{n}$ denote a Riemannian $n$-manifold with Laplacian operator $\Delta$. A smooth map $\phi: M^{n} \rightarrow \mathbb{E}^{N}$ of $M^{n}$ into the Euclidean $N$-space is said to be of finite type if it admits a finite spectral resolution:

$$
\phi=c+\sum_{t=1}^{k} \phi_{t}
$$

where $c$ is a constant vector in $\mathbb{E}^{N}, \phi_{t}$ 's are non-constant $\mathbb{E}^{N}$-valued maps such that $\Delta \phi_{t}=\lambda_{p_{t}} \phi_{t}$ with $\lambda_{p_{1}}<\lambda_{p_{2}}<\cdots<\lambda_{p_{k}}$. Otherwise, it is said to be of infinite type. When the spectral resolution (1.1) contains exactly $k$ non-constant terms, the map $\phi$ is called of $k$-type (see $[6,7,8]$ for details).

Let $S^{N-1} \subset \mathbb{E}^{N}$ denote the unit hypersphere of $\mathbb{E}^{N}$ centered at the origin. A spherical finite type map $\phi: M \rightarrow S^{N-1} \subset \mathbb{E}^{N}$ of a Riemannian manifold $M$ into $S^{N-1}$ is called mass-symmetric if the vector $c$ in its spectral resolution is the center of $S^{N-1}$ (which is the origin of $\mathbb{E}^{N}$ ). Otherwise, $\phi$ is called nonmass-symmetric. When $M$ is compact, $\phi$ is mass-symmetric if and only if we have $\int_{M} \phi * 1=0$.

Received August 30, 2005.

2000 Mathematics Subject Classification. Primary 53C40, 53C42, 53B25.

Key words and phrases. spherical Gauss map, finite type map, Clifford minimal torus, Veronese surface, equilateral torus. 
Let $G(n, m)$ denote the Grassmannian consisting of linear $n$-subspaces of $\mathbb{E}^{m}$. Given an isometric immersion $\mathrm{x}: M^{n} \rightarrow \mathbb{E}^{m}$, the classical Gauss map $\nu^{c}: M^{n} \rightarrow G(n, m)$ associated with $\mathrm{x}$ is the map which carries each $p \in M^{n}$ to the linear $n$-subspace of $\mathbb{E}^{m}$ obtained by parallel displacement of the tangent space $T_{p} M^{n}$. Since $G(n, m)$ can be canonically imbedded in $\wedge^{n} \mathbb{E}^{m}=\mathbb{E}^{\left(\begin{array}{c}m \\ n\end{array}\right)}$, the classical Gauss map gives rise to a well-defined map from $M^{n}$ into the Euclidean $\left(\begin{array}{c}m \\ n\end{array}\right)$-space $\mathbb{E}^{\left(\begin{array}{c}m \\ n\end{array}\right)}$.

The study of Euclidean submanifolds with finite type classical Gauss map was initiated by the first author and Piccinni in [11]. Since then many geometers have studied such submanifolds (see, for instance, $[1,2,3,4,9,10,12,13,17]$ ).

On the other hand, for a spherical submanifold $M^{n}$ in $S^{m-1}$, Obata studied in [19] the map which carries $p \in M^{n}$ to the totally geodesic $n$-sphere of $S^{m-1}$ determined by the tangent space $T_{p} M^{n}$; he also computed the induced metric on $M^{n}$ via his map. Since a totally geodesic $n$-sphere $S^{n}$ of $S^{m-1}$ determines a unique linear $(n+1)$-space containing the totally geodesic $S^{n}$ in $\mathbb{E}^{m}$, Obata's map can be extended to a map $\hat{\nu}$ of $M^{n}$ into the Grassmannian $G(n+1, m)$ in a natural way, known as the spherical Gauss map. The composition $\tilde{\nu}$ of $\hat{\nu}$ followed by the natural inclusion of $G(n+1, m)$ in $\mathbb{E}^{\left(\begin{array}{c}m \\ n+1\end{array}\right)}$ is also called the spherical Gauss map.

The geometrical behavior of the classical and spherical Gauss maps are different. For instance, the classical Gauss map of every compact Euclidean submanifold is mass-symmetric; but the spherical Gauss map of a spherical compact submanifold is not mass-symmetric in general. It was believed that in the study of spherical submanifolds the spherical Gauss map is more relevant than the classical Gauss map (cf. for instance, [20]).

The main purpose of this article is to study spherical Gauss map of spherical submanifolds in the frame work of finite type theory. The main problem is

"To what extent does the type number of the spherical Gauss map determine the spherical submanifolds?"

In section 3, we provide some basic results on spherical Gauss map. As a by-product, we are able to extend a result of Lawson on minimal surfaces in spheres. In section 4, we classify spherical submanifolds with 1-type spherical Gauss map. This classification result implies that every isoparametric hypersurface of $S^{n+1}$ has 1-type spherical Gauss map. In sections 5 and 6 , we prove that the Veronese surface in $S^{4}$ is the only spherical minimal surface with masssymmetric 2-type spherical Gauss map and the equilateral minimal torus in $S^{5}$ is the only minimal spherical surfaces with non-mass-symmetric 2-type spherical Gauss map. The last section provides more results on spherical Gauss map of spherical minimal submanifolds.

\section{Preliminaries}

Let $M^{n}$ be a submanifold of a Riemannian manifold $\tilde{M}^{m}$, i.e., the Riemannian $n$-manifold $M^{n}$ is isometrically immersed in the Riemannian $m$-manifold 
$\tilde{M}^{m}$. If $\nabla$ and $\tilde{\nabla}$ denote the Levi-Civita connections of $M^{n}$ and $\tilde{M}^{m}$, then the Gauss and Weingarten formulas of $M^{n}$ in $\tilde{M}^{m}$ are given respectively by

$$
\begin{aligned}
& \tilde{\nabla}_{X} Y=\nabla_{X} Y+h(X, Y), \\
& \tilde{\nabla}_{X} \xi=-A_{\xi} X+D_{X} \xi
\end{aligned}
$$

for $X, Y$ tangent to $M^{n}$ and $\xi$ normal to $N$, where $h$ is the second fundamental form, $D$ the normal connection, and $A$ the shape operator. The second fundamental form $h$ and the shape operator $A$ are related by $g\left(A_{\xi} X, Y\right)=$ $\tilde{g}(h(X, Y), \xi)$.

The mean curvature vector is $H=(1 / n) \operatorname{tr} h$. The submanifold is called minimal (respectively, totally geodesic) if its mean curvature vector (respectively, second fundamental form) vanishes identically.

We choose a local field of orthonormal frame $e_{1}, \ldots, e_{n}, e_{n+1}, \ldots, e_{m}$ such that, restricted to $M^{n}$, the vectors $e_{1}, \ldots, e_{n}$ are tangent to $M$ and hence $e_{n+1}, \ldots, e_{m}$ are normal to $M^{n}$. We shall make use of the following convention on the ranges of indices unless mentioned otherwise:

$$
1 \leq A, B, C, \ldots \leq m ; 1 \leq i, j, k, \ldots \leq n ; n+1 \leq r, s, t, \ldots \leq m .
$$

Put $\tilde{\nabla}_{X} e_{i}=\sum_{A} \omega_{i}^{A}(X) e_{A}$ and $\tilde{\nabla}_{X} e_{r}=\sum_{A} \omega_{r}^{A}(X) e_{A}$. With respect to the frame field of $M^{n}$ chosen above, let $\omega^{1}, \ldots, \omega^{n}, \omega^{n+1}, \ldots, \omega^{m}$ be the field of dual frame. Then the structure equations are given by

$$
\begin{aligned}
& d \omega^{A}=-\sum_{B} \omega_{B}^{A} \wedge \omega^{B}, \omega_{B}^{A}=-\omega_{A}^{B}, \\
& d \omega_{B}^{A}=-\sum_{C} \omega_{C}^{A} \wedge \omega_{B}^{C}+\Phi_{B}^{A}, \\
& \Phi_{B}^{A}=\frac{1}{2} \sum K_{B C D^{A}}^{A} \wedge \omega^{D}, K_{B C D}^{A}+K_{B D C}^{A}=0 .
\end{aligned}
$$

Restricting these forms to $M^{n}$ we get $\omega^{r}=0$. Since

$$
0=d \omega^{r}=-\sum_{i} \omega_{i}^{r} \wedge \omega^{i},
$$

Cartan's lemma yields $\omega_{i}^{r}=\sum_{j} h_{i j}^{r} \omega^{j}$ with $h_{i j}^{r}=h_{j i}^{r}$. The exterior differentiation of $\omega_{i}^{r}=\sum_{j} h_{i j}^{r}$ gives the equation of Codazzi:

$$
\begin{aligned}
& h_{i j ; k}^{r}=h_{i k ; j}^{r}, \\
& h_{i j ; k}^{r}=e_{k} h_{i j}^{r}-\sum_{\ell}\left(h_{i \ell}^{r} \omega_{j}^{\ell}\left(e_{k}\right)+h_{j \ell}^{r} \omega_{i}^{\ell}\left(e_{k}\right)\right)+\sum_{s} h_{i j}^{s} \omega_{s}^{r}\left(e_{k}\right) .
\end{aligned}
$$

From these we have the following Cartan's structure equations:

$$
\begin{aligned}
& d \omega^{i}=-\sum_{j} \omega_{j}^{i} \wedge \omega^{j}, d \omega_{j}^{i}=-\sum_{k} \omega_{k}^{i} \wedge \omega_{j}^{k}+\Omega_{j}^{i}, \\
& \Omega_{j}^{i}=\frac{1}{2} \sum_{k, \ell} R_{j k \ell}^{i} \omega^{k} \wedge \omega^{\ell}, \quad R_{j k \ell}^{i}=K_{j k \ell}^{i}+\sum_{r}\left(h_{i k}^{r} h_{j \ell}^{r}-h_{i \ell}^{r} h_{j k}^{r}\right) .
\end{aligned}
$$


The Ricci tensor $R_{j k}$ and the scalar curvature $S$ of $M^{n}$ are defined respectively by $R_{j k}=\sum_{i} R_{j i k}^{i}$ and $S=\sum_{i} R_{i i}$. If the ambient space $\tilde{M}^{m}$ is the Euclidean $m$-space $\mathbb{E}^{m}$, then (2.5) implies that the scalar curvature $S$ of $M^{n}$ satisfies

$$
S=n^{2}|H|^{2}-\|h\|^{2},
$$

where $|H|^{2}$ and $\|h\|^{2}$ are the squared norm of the mean curvature vector and of the second fundamental form of $M^{n}$ in $\mathbb{E}^{m}$. In particular, if $M^{n}$ is immersed in the unit hypersphere $S^{m-1}$, then (2.6) yields

$$
S=n(n-1)+n^{2}|\hat{H}|^{2}-\|\hat{h}\|^{2},
$$

where $\hat{H}$ and $\hat{h}$ are the mean curvature vector and the second fundamental form of $M^{n}$ in $S^{m-1}$, respectively. For $M^{n}$ in $S^{m-1}$, we also have (cf. [5])

$$
H=\hat{H}-\mathbf{x}, \quad h(X, Y)=\hat{h}(X, Y)-g(X, Y) \mathbf{x} .
$$

A submanifold $M$ is called isotropic if, at each given point $p \in M$, the length $|h(u, u)|$ is independent of the choice of the unit tangent vector $u \in T_{p} M$.

Theorem 2.1. [11] Let $\phi: M \rightarrow \mathbb{E}^{N}$ be a smooth map from a compact Riemannian manifold $M$ into the Euclidean $N$-space and let $\tau:=\operatorname{div}(d \phi)$ be the tension field of $\phi$. Then we have:

(i) $\phi$ is of finite type if and only if there is a non-trivial polynomial $Q(t)$ satisfying $Q(\Delta) \tau=0$.

(ii) If $\phi$ is of finite type, there is a unique monic polynomial $P(t)$ of least degree, called the minimal polynomial, which satisfies $P(\Delta) \tau=0$.

(iii) If $\phi$ is of finite type, then $\phi$ is of k-type if and only if the minimal polynomial $P$ is of degree $k$.

The same results hold if $\tau$ is replaced by $\phi-\phi_{0}$ with

$$
\phi_{0}=\left(\int_{M} \phi * 1\right) /(\operatorname{vol}(M)) \text {. }
$$

\section{Some basic results on spherical Gauss map}

Let $V$ be an oriented $k$-plane in $\mathbb{E}^{m}$. If $\epsilon_{1}, \ldots, \epsilon_{k}$ is an oriented orthonormal basis of $V$, then $\epsilon_{1} \wedge \cdots \wedge \epsilon_{k}$ is a decomposable $k$-vector of norm one which gives $\epsilon_{1} \wedge \cdots \wedge \epsilon_{k}$ the orientation of $V$. Conversely, each decomposable $k$-vector of norm one determines a uniquely $k$-plane in $\mathbb{E}^{m}$. Hence, if $G(k, m)$ denotes the Grassmannian of oriented $k$-planes in $\mathbb{E}^{m}$, then $G(k, m)$ can be identified naturally with the decomposable $k$-vectors of norm one in $\mathbb{E}^{\left(\begin{array}{c}m \\ k\end{array}\right)}=\wedge^{k} \mathbb{E}^{m}$. In

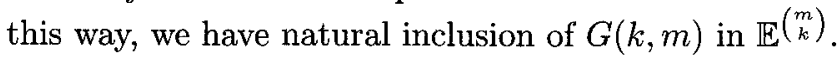

In this article, we shall always regard $S^{m-1}$ as the unit hypersphere of $\mathbb{E}^{m}$ centered at the origin. Moreover, for an isometric immersion $\mathbf{x}$ of a Riemannian $n$-manifold $M^{n}$ into $S^{m-1}$, we identify each tangent vector $v \in T M^{n}$ with the its image $d \mathbf{x}(v)$ under the differential $d \mathbf{x}$. 
For each point $p \in M^{n}$, let $e_{1}, \ldots, e_{n}$ be an orthonormal basis of $T_{p} M^{n}$. Then the $n+1$ vectors $\mathbf{x}, e_{1}, \ldots, e_{n}$ determine a linear $(n+1)$-subspace in $\mathbb{E}^{m}$. The intersection of this linear subspace and $S^{m-1}$ is the totally geodesic $n$-sphere determined by $T_{p} M^{n}$ as in [19]. Thus, for a spherical immersion $\mathbf{x}: M^{n} \rightarrow S^{m-1}$, Obata's map can be considered as:

$$
\nu: M^{n} \rightarrow G(n+1, m)
$$

which carries each $p \in M^{n}$ to $\nu(p)=\mathbf{x} \wedge e_{1} \wedge \cdots \wedge e_{n}$.

Let $S^{\left(\begin{array}{c}m \\ n+1\end{array}\right)-1}$ be the unit hypersphere in $\mathbb{E}^{\left(\begin{array}{c}m \\ n+1\end{array}\right)}$ centered at the origin. Then $G(n+1, m)$ is a submanifold of $S^{\left(\begin{array}{c}m \\ n+1\end{array}\right)-1}$, which gives the natural inclusion:

$$
\iota: G(n+1, m) \subset S^{\left(\begin{array}{c}
m \\
n+1
\end{array}\right)-1} \subset \mathbb{E}^{\left(\begin{array}{c}
m \\
n+1
\end{array}\right)} .
$$

The spherical Gauss map $\hat{\nu}: M^{n} \rightarrow E^{\left(\begin{array}{c}m \\ n+1\end{array}\right)}$ associated with $\mathbf{x}$ is thus given by

$$
\tilde{\nu}=\mathbf{x} \wedge e_{1} \wedge \cdots \wedge e_{n}: M^{n} \rightarrow G(n+1, m) \subset S^{\left(\begin{array}{c}
m \\
n+1
\end{array}\right)-1} \subset \mathbb{E}^{\left(\begin{array}{c}
m \\
n+1
\end{array}\right)} .
$$

The associated map $\hat{\nu}$ of $M^{n}$ in $S^{\left(\begin{array}{c}m \\ n+1\end{array}\right)-1}$ is also called the spherical Gauss map.

Let $M$ be a Riemannian manifold and $G$ a closed subgroup of the group $I(M)$ of isometries which acts transitively on $M$. An immersion $f$ of $M$ into another Riemannian manifold $\tilde{M}$ is called $G$-equivariant if there exists a homomorphism $\zeta: G \rightarrow I(\tilde{M})$ such that $f(a(p))=\zeta(a) f(p)$ for each $a \in G$ and $p \in M$.

An important consequence of Theorem 2.1 is the following finiteness result.

Proposition 3.1. If $\mathrm{x}: M \rightarrow S^{m-1} \subset \mathbb{E}^{m}$ is an equivariant isometric immersion of a compact homogeneous Riemannian n-manifold into $S^{m-1}$, then its spherical Gauss map $\tilde{\nu}$ is of finite type. Moreover, the type number of $\tilde{\nu}$ is at most $\left(\begin{array}{c}m \\ n+1\end{array}\right)$.

Proof. Let $\tau$ be the tension field of $\tilde{\nu}$. Then $\tau, \Delta \tau, \ldots, \Delta{ }^{\left(\begin{array}{c}m \\ n+1\end{array}\right)} \tau$ are linearly dependent at a given point $u \in M$. Thus there is a polynomial $Q(t)$ of degree $\leq N$ satisfying $Q(\Delta) \tau=0$ at $u$. Since $\mathbf{x}$ is equivariant, the group of isometries of the Euclidean space acts transitively on $M$ as well as on the tangent bundle of $M$. Hence, it acts transitively on its spherical Gauss of map. Thus, we have $Q(\Delta) \tau=0$ at each point in $M$. Therefore, Theorem 2.1 implies that the spherical Gauss map is of finite type. Since the degree of the minimal polynomial of $Q$ is $\leq\left(\begin{array}{c}m \\ n+1\end{array}\right)$, the type number is at most $\left(\begin{array}{c}m \\ n+1\end{array}\right)$ according to theorem 2.1.

Remark 3.1. Proposition 3.1 shows that there exist abundant examples of nice spherical submanifolds with finite type spherical Gauss map.

By differentiating $\tilde{\nu}$ in $(3.2)$ we find

$$
e_{j} \tilde{\nu}=\sum_{r, k} h_{j k}^{r} \mathbf{x} \wedge e_{1} \wedge \cdots \wedge e_{k-1} \wedge e_{r} \wedge e_{k+1} \wedge \cdots \wedge e_{n} .
$$


From (3.3) and Gauss' equation, we see that the induced metric $\hat{g}$ on $M^{n}$ via $\hat{\nu}$ is

$$
\hat{g}=\sum_{j, k}\left\{(n-1) \delta_{j k}+\sum_{r}\left(\operatorname{tr} h^{r}\right) h_{j k}^{r}-R_{j k}\right\} \omega^{j} \otimes \omega^{k} .
$$

Since the Laplacian of $\hat{\nu}$ is defined by

$$
\Delta \tilde{\nu}=-\sum_{i} e_{i} e_{i} \tilde{\nu}+\left(\nabla_{e_{i}} e_{i}\right) \tilde{\nu}
$$

we obtain from (3.3) that

$$
\begin{aligned}
\Delta \tilde{\nu}= & n H \wedge e_{1} \wedge \cdots \wedge e_{n}+\|h\|^{2} \tilde{\nu} \\
& -n \sum_{k} \mathbf{x} \wedge e_{1} \wedge \cdots \wedge e_{k-1} \wedge D_{e_{k}} \hat{H} \wedge e_{k+1} \wedge \cdots \wedge e_{n} \\
& -\sum_{r, s, i, j, k} h_{i j}^{r} h_{i k}^{s} \mathbf{x} \wedge e_{1} \wedge \cdots \wedge \underbrace{e_{s}}_{k-t h} \wedge \cdots \wedge \underbrace{e_{r}}_{j-t h} \wedge \cdots \wedge e_{n} .
\end{aligned}
$$

By applying (3.6), (2.8) and the last equation in (2.7), we obtain

$$
\begin{aligned}
\Delta \tilde{\nu}= & \|\hat{h}\|^{2} \tilde{\nu}+n \hat{H} \wedge e_{1} \wedge \cdots \wedge e_{n} \\
& -n \sum_{k} \mathbf{x} \wedge e_{1} \wedge \cdots \wedge e_{k-1} \wedge D_{e_{k}} \hat{H} \wedge e_{k+1} \wedge \cdots \wedge e_{n} \\
& +\sum_{r, s, j, k} R_{s j k}^{r} \mathbf{x} \wedge e_{1} \wedge \cdots \wedge \underbrace{e_{s}}_{k-t h} \wedge \cdots \wedge \underbrace{e_{r}}_{j-t h} \wedge \cdots \wedge e_{n} .
\end{aligned}
$$

A map between two Riemannian manifolds is called harmonic if its tension field, $\tau=\operatorname{div}(d \phi)$, vanishes identically. For an isometric immersion, the tension field and the mean curvature vector field differ only by the dimension of the submanifold.

In [21], Ruh and Vilms proved that a Euclidean submanifold has parallel mean curvature vector if and only if its classical Gauss map is harmonic. On the other hand, for spherical Gauss map we have the following.

Proposition 3.2. Let $\mathbf{x}:\left(M^{n}, g\right) \rightarrow S^{m-1}$ be an isometric immersion of a Riemannian $n$-manifold. Then we have:

(a) Obata's map $\nu:\left(M^{n}, g\right) \rightarrow G(n+1, m)$ is a harmonic map if and only if $\mathbf{x}:\left(M^{n}, g\right) \rightarrow S^{m-1}$ is a minimal immersion;

(b) The spherical Gauss map $\tilde{\nu}:\left(M^{n}, g\right) \rightarrow \mathbb{E}^{\left(\begin{array}{c}m \\ n+1\end{array}\right)}$ is harmonic if and only if $\mathbf{x}:\left(M^{n}, g\right) \rightarrow S^{m-1}$ is totally geodesic.

Proof. Statement (a) follows from (3.7) and the fact that decomposable $(n+1)-$ vectors of the forms:

$$
e_{r} \wedge e_{1} \wedge \cdots \wedge e_{n}, \mathbf{x} \wedge e_{1} \wedge \cdots \wedge e_{r} \wedge \cdots \wedge e_{n}
$$

provide a basis for the tangent space of $G(n+1, m)$. Statement (b) is an immediate consequence of (3.7). 
Lawson proved in [18] that the spherical Gauss map of minimal surfaces in $S^{3}$ are minimal surfaces in $S^{3}$ (possibly with branched points). An immediate application of Proposition 3.2 is the following extension of Lawson's result.

Proposition 3.3. Let $\mathbf{x}:\left(M^{2}, g\right) \rightarrow S^{m-1}$ be a minimal surface without totally geodesic points. Then the Obata map $\nu:\left(M^{2}, \hat{g}\right) \rightarrow G(3, m)$ is a minimal isometric immersion (with respect to the induced metric $\hat{g}$ ).

Proof. For the minimal isometric immersion $x:\left(M^{2}, \hat{g}\right) \rightarrow S^{m-1},(3.4)$ reduces to

$$
\hat{g}=(1-K) g,
$$

where $K$ is the Gauss curvature of $\left(M^{2}, g\right)$. Since harmonicity is preserved under conformal change of metric, Proposition 3.2 implies that Obata's map $\nu$ is harmonic. Thus this immersion is a minimal immersion, since it is isometric.

\section{Spherical submanifolds with 1-type spherical Gauss map}

In this section, we completely classify spherical submanifolds with 1-type spherical Gauss map.

Theorem 4.1. A submanifold of $S^{m-1}$ has mass-symmetric 1-type spherical Gauss map if and only if it is a minimal submanifold of $S^{m-1}$ with constant scalar curvature and flat normal connection.

Proof. If the spherical submanifold has mass-symmetric 1-type spherical Gauss map $\tilde{\nu}$, the $\Delta \tilde{\nu}$ and $\tilde{\nu}$ are proportional. Since the second and third terms on the right hand side of (3.7) are perpendicular to $\tilde{\nu}$, we see from (3.7) that $\tilde{\nu}$ is mass-symmetric 1-type if and only if $\hat{H}=R_{s j k}^{r}=0$ and $\|\hat{h}\|^{2}$ is constant. Hence, by applying this and (2.7) we obtain the desired result.

The standard imbedding of $S^{1}\left(\frac{1}{\sqrt{2}}\right) \times S^{1}\left(\frac{1}{\sqrt{2}}\right)$ in $S^{3}$ is called Clifford minimal torus.

Theorem 4.2. A non-totally geodesic surface in $S^{m-1}$ has mass-symmetric 1-type spherical Gauss map if and only if it is an open portion of the Clifford minimal torus (lying fully in a totally geodesic 3-sphere $S^{3} \subset S^{m-1}$ ).

Proof. It is easy to verify that the spherical Gauss map of the Clifford minimal torus satisfies $\Delta \tilde{\nu}=2 \tilde{\nu}$. Thus, it has mass-symmetric 1-type spherical Gauss map. The converse follows from Theorem 4.1 and the fact that the only minimal surfaces of $S^{m-1}$ with constant Gauss curvature and flat normal connection are open portions of a totally geodesic 2-sphere or of the Clifford minimal torus.

Theorem 4.3. An n-dimensional submanifold of $S^{m-1}$ has non-mass-symmetric 1-type spherical Gauss map if and only if it has constant scalar curvature and it is immersed in a totally geodesic $(n+1)$-sphere $S^{n+1} \subset S^{m-1}$ as a hypersurface with nonzero constant mean curvature. 
Proof. Let $\mathbf{x}: M^{n} \rightarrow S^{m-1}$ be an isometric immersion of a Riemannian $n$ manifold into $S^{m-1}$. If the spherical Gauss map $\tilde{\nu}$ of $\mathbf{x}$ is non-mass-symmetric 1-type, then we have $\Delta \tilde{\nu}=\lambda_{p}(\tilde{\nu}-c)$ for some vector $c$ and real number $\lambda_{p}$. Thus we have

$$
(\Delta \tilde{\nu})_{j}=\lambda_{p}(\tilde{\nu})_{j}
$$

where $(\cdot)_{j}=e_{j}(\cdot)$.

On the other hand, by a direct long computation, we obtain from (3.7) that (4.2)

$$
\begin{aligned}
& e_{i}(\Delta \tilde{\nu}) \\
& =\|\hat{h}\|_{i}^{2} \tilde{\nu}+\|\hat{h}\|^{2} \sum_{r, k} h_{i k}^{r} \mathbf{x} \wedge e_{1} \wedge \cdots \wedge \underbrace{e_{r}}_{k-t h} \wedge \cdots \wedge e_{n} \\
& +2 n D_{e_{i}} \hat{H} \wedge e_{1} \wedge \cdots \wedge e_{n}+n \sum_{r, k} h_{i k}^{r} \hat{H} \wedge e_{1} \wedge \cdots \wedge \underbrace{e_{r}}_{k-t h} \wedge \cdots \wedge e_{n} \\
& -n \sum_{j \neq k} \omega_{j}^{k}\left(e_{i}\right) \mathbf{x} \wedge e_{1} \wedge \cdots \wedge e_{j-1} \wedge \underbrace{e_{k}}_{j-t h} \wedge e_{j+1} \wedge \cdots \wedge \underbrace{D_{e_{k}} \hat{H}}_{k-t h} \wedge \cdots \wedge e_{n} \\
& -n \sum_{j \neq k} h_{i j}^{r} \mathbf{x} \wedge e_{1} \wedge \cdots \wedge e_{j-1} \wedge \underbrace{e_{r}}_{j-t h} \wedge e_{j+1} \wedge \cdots \wedge \underbrace{D_{e_{k}} \hat{H}}_{k-t h} \wedge \cdots \wedge e_{n} \\
& +n \sum\left\langle A_{D_{e_{k}} \hat{H}} e_{i}, e_{k}\right\rangle \tilde{\nu}-n \sum_{k} \mathbf{x} \wedge e_{1} \wedge \cdots \wedge \underbrace{D_{e_{i}} D_{e_{k}} \hat{H}}_{k-t h} \wedge \cdots \wedge e_{n} \\
& +\sum_{r, s, j, k}\left(\left(e_{i} R_{s j k}^{r}\right) \mathbf{x}+R_{s j k}^{r} e_{i}\right) \wedge e_{1} \wedge \cdots \wedge \underbrace{e_{s}}_{k-t h} \wedge \cdots \wedge \underbrace{e_{r}}_{j-t h} \wedge \cdots \wedge e_{n} \\
& +\sum_{r, s} \sum_{j, k, \ell \neq} R_{s j k}^{r}\{h_{i \ell}^{t} \mathbf{x} \wedge e_{1} \wedge \cdots \wedge \underbrace{\ell-t h} \wedge \cdots \wedge \underbrace{e_{s}}_{k-t h} \wedge \cdots \wedge \underbrace{e_{r}}_{j-t h} \wedge \cdots \wedge e_{n} \\
& +\omega_{\ell}^{h}\left(e_{i}\right) \mathbf{x} \wedge e_{1} \wedge \cdots \wedge \underbrace{\ell-t h} \wedge \cdots \wedge \underbrace{e_{s}}_{k-t h} \wedge \cdots \wedge \underbrace{e_{r}}_{j-t h} \wedge \cdots \wedge e_{n}\} \\
& +\sum_{r, s, j, k}\left(R_{s j k}^{r} \omega_{s}^{t}\left(e_{i}\right)-R_{s j k}^{t} \omega_{s}^{r}\left(e_{i}\right)\right) \mathbf{x} \wedge e_{1} \wedge \cdots \wedge \underbrace{e_{t}}_{k-t h} \wedge \cdots \wedge \underbrace{e_{r}}_{j-t h} \wedge \cdots \wedge e_{n} .
\end{aligned}
$$

Case (a): $\hat{H}=0$. In this case, equation (4.2) reduces to

$$
\begin{aligned}
& e_{i}(\Delta \tilde{\nu}) \\
= & \|\hat{h}\|_{i}^{2} \tilde{\nu}+\|\hat{h}\|^{2} \sum_{r, k} h_{i k}^{r} \mathbf{x} \wedge e_{1} \wedge \cdots \wedge \underbrace{e_{r}}_{k-t h} \wedge \cdots \wedge e_{n} \\
& +\sum_{r, s, j, k}\left(\left(e_{i} R_{s j k}^{r}\right) \mathbf{x}+R_{s j k}^{r} e_{i}\right) \wedge e_{1} \wedge \cdots \wedge \underbrace{e_{s}}_{k-t h} \wedge \cdots \wedge \underbrace{e_{r}}_{j-t h} \wedge \cdots \wedge e_{n}
\end{aligned}
$$




$$
\begin{aligned}
& +\sum_{r, s} \sum_{j, k, \ell \neq} R_{s j k}^{r}\{h_{i \ell}^{t} \mathbf{x} \wedge e_{1} \wedge \cdots \wedge \underbrace{e_{t}}_{\ell-t h} \wedge \cdots \wedge \underbrace{e_{s}}_{k-t h} \wedge \cdots \wedge \underbrace{e_{r}}_{j-t h} \wedge \cdots \wedge e_{n} \\
& +\sum_{h} \omega_{\ell}^{h}\left(e_{i}\right) \mathbf{x} \wedge e_{1} \wedge \cdots \wedge \underbrace{e_{h}}_{\ell-t h} \wedge \cdots \wedge \underbrace{e_{s}}_{k-t h} \wedge \cdots \wedge \underbrace{e_{r}}_{j-t h} \wedge \cdots \wedge e_{n}\} \\
& +\sum_{r, s, j, k}\left(R_{s j k}^{r} \omega_{s}^{t}\left(e_{i}\right)-R_{s j k}^{t} \omega_{s}^{r}\left(e_{i}\right)\right) \mathbf{x} \wedge e_{1} \wedge \cdots \wedge \underbrace{e_{t}}_{k-t h} \wedge \cdots \wedge \underbrace{e_{r}}_{j-t h} \wedge \cdots \wedge e_{n} .
\end{aligned}
$$

By comparing (3.3), (4.1) and (4.3), we get $\|\hat{h}\|_{i}^{2}=R_{s j k}^{r}=0$. Thus, $M^{n}$ has constant scalar curvature and flat normal connection. So, Theorem 4.1 implies that $\tilde{\nu}$ is mass-symmetric 1-type. This is a contradiction.

Case (b); $\hat{H} \neq 0$. Since the term $D_{e_{i}} \hat{H} \wedge e_{1} \wedge \cdots \wedge e_{n}$ appears only in $\tilde{\nabla}_{e_{i}}(\Delta \tilde{\nu})$ of (4.2), not in $e_{i}(\tilde{\nu})$, we know from $(3.3),(4.1)$ and (4.2) that $D \hat{H}=0$. Thus, $M^{n}$ has parallel nonzero mean curvature vector in $S^{m-1}$. So, it has nonzero constant mean curvature. Therefore, equation (4.2) reduces to

$$
\begin{aligned}
& e_{i}(\Delta \tilde{\nu}) \\
= & n \sum_{r, k} h_{i k}^{r} \hat{H} \wedge e_{1} \wedge \cdots \wedge e_{k-1} \wedge e_{r} \wedge e_{k+1} \wedge \cdots \wedge e_{n} \\
& +\|\hat{h}\|_{i}^{2} \tilde{\nu}+\|\hat{h}\|^{2} \sum_{r, k} h_{i k}^{r} \mathbf{x} \wedge e_{1} \wedge \cdots \wedge e_{k-1} \wedge e_{r} \wedge e_{k+1} \wedge \cdots \wedge e_{n} \\
& +\sum_{r, s, j, k}\left\{\left(e_{i} R_{s j k}^{r}\right) \mathbf{x}+R_{s j k}^{r} e_{i}\right\} \wedge e_{1} \wedge \cdots \wedge \underbrace{e_{s}}_{k-t h} \wedge \cdots \wedge \underbrace{e_{r}}_{j-t h} \wedge \cdots \wedge e_{n} \\
& +\sum_{r, s} \sum_{j, k, \ell \neq} R_{s j k}^{r}\{h_{i \ell}^{t} \mathbf{x} \wedge e_{1} \wedge \cdots \wedge \underbrace{e_{t}}_{\ell-t h} \wedge \cdots \wedge \underbrace{e_{s}}_{k-t h} \wedge \cdots \wedge \underbrace{e_{r}}_{j-t h} \wedge \cdots \wedge e_{n} \\
& +\omega_{\ell}^{h}\left(e_{i}\right) \mathbf{x} \wedge e_{1} \wedge \cdots \wedge \underbrace{e_{h}}_{\ell-t h} \wedge \cdots \wedge \underbrace{e_{s}}_{k-t h} \wedge \cdots \wedge \underbrace{e_{r}}_{j-t h} \wedge \cdots \wedge e_{n}^{\left.e_{n}\right\}} \\
& +\sum_{r, s, j, k}\left(R_{s j k}^{r} \omega_{s}^{t}\left(e_{i}\right)-R_{s j k}^{t} \omega_{s}^{r}\left(e_{i}\right)\right) \mathbf{x} \wedge e_{1} \wedge \cdots \wedge \underbrace{e_{t}}_{k-t h} \wedge \cdots \wedge \underbrace{e_{r}}_{j-t h} \wedge \cdots \wedge e_{n} .
\end{aligned}
$$

From (3.3), (4.1) and (4.4) we know that $\|\hat{h}\|$ and scalar curvature are constant. Also, we have

$$
\begin{aligned}
& n \sum_{r, k} h_{i k}^{r} \hat{H} \wedge e_{1} \wedge \cdots \wedge e_{k-1} \wedge e_{r} \wedge e_{k+1} \wedge \cdots \wedge e_{n} \\
+ & \sum_{r, s, j, k} R_{s j k}^{r} e_{i} \wedge e_{1} \wedge \cdots \wedge \underbrace{e_{s}}_{k-t h} \wedge \cdots \wedge \underbrace{e_{r}}_{j-t h} \wedge \cdots \wedge e_{n}=0 .
\end{aligned}
$$

Put $\hat{H}=|\hat{H}| e_{n+1}$. It follows from (4.5) that $R_{s j k}^{r}=0$ for $r, s \geq n+2$ and $j, k=1, \ldots, n$. We also find $R_{s j k}^{3}=0$ from $D \hat{H}=0$. Thus, the normal 
connection of $M^{n}$ in $S^{m-1}$ is flat. Therefore, (4.5) yields

$$
\sum_{r=n+2}^{m-1} \sum_{k} h_{i k}^{r} e_{n+1} \wedge e_{1} \wedge \cdots \wedge e_{k-1} \wedge e_{r} \wedge e_{k+1} \wedge \cdots \wedge e_{n}=0 .
$$

We see from (4.6) that the first normal space $\operatorname{Im} h$ is spanned by $e_{n+1}$. Therefore, by the reduction theorem of Erbarcher, we conclude that $M^{n}$ is contained in a totally geodesic $S^{n+1} \subset S^{m-1}$.

Conversely, if $M^{n}$ has constant scalar curvature and it lies in a totally geodesic $S^{n+1} \subset S^{m-1}$ with nonzero constant mean curvature, then (4.4) reduces to

$$
e_{i}(\Delta \tilde{\nu})=\|\hat{h}\|^{2} \sum_{r, k} h_{i k}^{r} \mathbf{x} \wedge e_{1} \wedge \cdots \wedge e_{k-1} \wedge e_{r} \wedge e_{k+1} \wedge \cdots \wedge e_{n}
$$

From this we have $e_{i}(\Delta \tilde{\nu})=\|\hat{h}\|^{2} e_{i}(\tilde{\nu})$. Thus, $\Delta(\tilde{\nu}-c)=\|\hat{h}\|^{2} \tilde{\nu}$ for some nonzero vector $c$. Since $\|\hat{h}\|$ is constant, this shows that $\tilde{\nu}$ is non-mass-symmetric 1-type.

Combining Theorems 4.1 and 4.3 yields the following remarkable result.

Corollary 4.1. Every isoparametric hypersurface of $S^{n+1}$ has 1-type spherical Gauss map.

Remark 4.1. Theorem 4.1 and Theorem 4.3 determine completely spherical submanifolds with 1-type spherical Gauss map. On the other hand, Proposition 3.1, Theorem 4.1 and Theorem 4.3 imply that there exist rich examples of spherical submanifolds with higher type spherical Gauss map. In particular, most equivariant isometric immersions of compact homogeneous Riemannian spaces into $S^{m-1}$ have high finite type spherical Gauss map.

Remark 4.2. Another implication of Theorems 4.1 and 4.3 is that finite type Euclidean submanifolds (cf. [6]) and spherical submanifolds with finite type spherical Gauss map are quite different. For instance, a submanifold in $\mathbb{E}^{m}$ is of 1-type if and only if it is either a minimal submanifold of $\mathbb{E}^{m}$ or a minimal submanifold of a hypersphere of $\mathbb{E}^{m}$. On contrast, according to Theorems 4.1 and 4.3, most spherical minimal submanifolds (except those with constant scalar curvature and flat normal connection) have higher type spherical Gauss map. In the next two sections, we completely classify spherical minimal surfaces with 2-type spherical Gauss map.

\section{Veronese surface and its spherical Gauss map}

For a natural number $k$, the set of spherical harmonic polynomials of degree $k$ of three variables is a $(2 k+1)$-dimensional vector space. Consider the unit sphere $S^{2 k}$ in the vector space with the standard inner product. We have an isometric minimal immersion: $\psi_{k}: S^{2}(K) \rightarrow S^{2 k}$ of the 2-sphere $S^{2}(K)$ of curvature $K=2 /(k(k+1))$ into $S^{2 k}$, which is known as a Veronese-Borůvka 
sphere. The immersion $\psi_{2}: S^{2}\left(\frac{1}{3}\right) \rightarrow S^{4}$ is known as the Veronese surface. Wallach [22] proved that any minimal surface of positive constant curvature $K$ in a unit sphere is an open part of a Veronese-Borůvka sphere.

Let $(x, y, z)$ be the natural coordinate system of $\mathbb{E}^{3}$ and $\left(u_{1}, \ldots, u_{5}\right)$ that of $\mathbb{E}^{5}$. The minimal immersion $\psi_{2}$ of $S^{2}\left(\frac{1}{3}\right)$ into $S^{4} \subset \mathbb{E}^{5}$ can be expressed explicitly as

$$
\begin{gathered}
u_{1}=\frac{y z}{\sqrt{3}}, \quad u_{2}=\frac{x z}{\sqrt{3}}, \quad u_{3}=\frac{x y}{\sqrt{3}}, \quad u_{4}=\frac{x^{2}-y^{2}}{2 \sqrt{3}}, \\
u_{5}=\frac{1}{6}\left(x^{2}+y^{2}-2 z^{2}\right)
\end{gathered}
$$

Theorem 4.1 implies that a totally geodesic $S^{2}$ in $S^{3}$ has 1-type spherical Gauss map. The next result provides a very simple characterization of Veronese surface in terms of 2-type spherical Gauss map.

Theorem 5.1. A minimal surface $M$ of $S^{m-1}$ is an open portion of the Veronese surface (lying fully in a totally geodesic $S^{4} \subset S^{m-1}$ ) if and only if it has mass-symmetric 2-type spherical Gauss map.

Proof. First, assume that $M^{2}$ is an open portion of the Veronese surface defined by (5.1). If we choose the spherical coordinates:

$$
\begin{gathered}
x=\sqrt{3} \sin \left(\frac{u}{\sqrt{3}}\right) \cos \left(\frac{v}{\sqrt{3}}\right), y=\sqrt{3} \sin \left(\frac{u}{\sqrt{3}}\right) \sin \left(\frac{v}{\sqrt{3}}\right), \\
z=\sqrt{3} \cos \left(\frac{u}{\sqrt{3}}\right)
\end{gathered}
$$

on $S^{2}(\sqrt{3})$, then the metric tensor $g$ and the Laplacian operator are

$$
\begin{aligned}
& g=d u^{2}+\sin ^{2}\left(\frac{u}{\sqrt{3}}\right) d v^{2} \\
& \Delta=\frac{1}{\sqrt{3}} \cot \left(\frac{u}{\sqrt{3}}\right) \frac{\partial}{\partial u}+\frac{\partial^{2}}{\partial u^{2}}+\csc ^{2}\left(\frac{u}{\sqrt{3}}\right) \frac{\partial^{2}}{\partial v^{2}} .
\end{aligned}
$$

Let us choose

$$
e_{1}=\frac{\partial}{\partial u}, e_{2}=\csc \left(\frac{u}{\sqrt{3}}\right) \frac{\partial}{\partial v}, \hat{h}\left(e_{1}, e_{1}\right)=\frac{e_{3}}{\sqrt{3}}, \hat{h}\left(e_{1}, e_{2}\right)=\frac{e_{4}}{\sqrt{3}} .
$$

Then $e_{1}, e_{2}, e_{3}, e_{4}$ form an orthonormal frame field. Moreover, we have

$$
\begin{aligned}
& h_{11}^{3}=h_{12}^{4}=\frac{1}{\sqrt{3}}, \quad h_{12}^{3}=h_{11}^{4}=-h_{22}^{4}=0, \\
& \omega_{3}^{4}=2 \omega_{1}^{2}=\frac{2}{\sqrt{3}} \cot \left(\frac{u}{\sqrt{3}}\right) \omega^{2}, \quad\|\hat{h}\|^{2}=-2 K^{D}=\frac{4}{3} .
\end{aligned}
$$

By applying (3.7), (5.6) and a long computation we find

$$
\Delta \tilde{\nu}=\frac{4}{3} \tilde{\nu}-\frac{4}{3} \mathbf{x} \wedge e_{3} \wedge e_{4}, \Delta^{2} \tilde{\nu}=\frac{32}{3} \tilde{\nu}-\frac{56}{3} \mathbf{x} \wedge e_{3} \wedge e_{4}
$$

Thus, if we put

$$
\tilde{\nu}_{1}=\frac{4}{5} \tilde{\nu}+\frac{2}{5} \mathbf{x} \wedge e_{3} \wedge e_{4}, \quad \tilde{\nu}_{3}=\frac{1}{5} \tilde{\nu}-\frac{2}{5} \mathbf{x} \wedge e_{3} \wedge e_{4},
$$


we obtain $\tilde{\nu}=\tilde{\nu}_{1}+\tilde{\nu}_{3}$ with $\Delta \tilde{\nu}_{1}=\frac{2}{3} \tilde{\nu}_{1}$, and $\Delta \tilde{\nu}_{3}=4 \tilde{\nu}_{3}$, which implies that the spherical Gauss map is mass-symmetric and of 2-type.

Let us use the following convention on the ranges of indices:

$$
1 \leq i, j, k, \ell \leq 2 ; 3 \leq r, s, t \leq 4 ; 5 \leq \alpha, \beta, \gamma \leq m-1 .
$$

Next, assume that $\mathrm{x}: M^{2} \rightarrow S^{m-1}$ is a minimal surface with mass-symmetric 2-type spherical Gauss map. We choose $e_{n+1}, \ldots, e_{m-1}, e_{m}$ in such way that

$$
e_{m}=\mathbf{x}, \quad A_{e_{5}}=\cdots=A_{e_{m-1}}=0 .
$$

Then $A_{r_{m}}=-I$ and $R_{s 12}^{r}=0,(r, s) \neq(3,4),(4,3)$. Thus, we get from $(3.7)$ that

$$
\begin{aligned}
& \Delta \tilde{\nu}=\|\hat{h}\|^{2} \tilde{\nu}+2 K^{D} \times \wedge e_{3} \wedge e_{4}, \\
& K^{D}=\sum_{j}\left(h_{2 j}^{3} h_{1 j}^{4}-h_{1 j}^{3} h_{2 j}^{4}\right),
\end{aligned}
$$

and

$$
\begin{aligned}
\Delta^{2} \tilde{\nu}= & \left(\Delta\|\hat{h}\|^{2}+\|\hat{h}\|^{4}\right) \tilde{\nu}+2\left(K^{D}\|\hat{h}\|^{2}+\Delta K^{D}\right) \mathbf{x} \wedge e_{3} \wedge e_{4} \\
& +2 K^{D} \Delta\left(\mathbf{x} \wedge e_{3} \wedge e_{4}\right)-2 \sum\|\hat{h}\|_{j}^{2} \tilde{\nabla} e_{e_{j}} \tilde{\nu} \\
& -4 \sum K_{j}^{D} \tilde{\nabla}_{e_{j}}\left(\mathbf{x} \wedge e_{3} \wedge e_{4}\right)
\end{aligned}
$$

where $\|\hat{h}\|_{j}^{2}=e_{j}\|\hat{h}\|^{2}$ and $K_{j}^{D}=e_{j} K^{D}$.

From (2.1) we have

$$
\begin{aligned}
e_{j}\left(\mathbf{x} \wedge e_{3} \wedge e_{4}\right)= & e_{j} \wedge e_{3} \wedge e_{4}-\sum_{i} h_{i j}^{3} \mathbf{x} \wedge e_{i} \wedge e_{4} \\
& -\sum_{i} h_{i j}^{4} \mathbf{x} \wedge e_{3} \wedge e_{i}+\sum_{\alpha} \omega_{3}^{\alpha}\left(e_{j}\right) \mathbf{x} \wedge e_{\alpha} \wedge e_{4} \\
& +\sum_{\alpha} \omega_{4}^{\alpha}\left(e_{j}\right) \mathbf{x} \wedge e_{3} \wedge e_{\alpha} .
\end{aligned}
$$

Moreover, we obtain from (2.3) and (5.12) that

$$
\begin{aligned}
& \Delta\left(\mathbf{x} \wedge e_{3} \wedge e_{4}\right) \\
= & 2 K^{D} \tilde{\nu} \\
& +\left(2+\|\hat{h}\|^{2}+\sum_{\alpha}\left\|\omega_{3}^{\alpha}\right\|^{2}+\sum_{\alpha}\left\|\omega_{4}^{\alpha}\right\|^{2}\right) \mathbf{x} \wedge e_{3} \wedge e_{4} \\
& -2 \sum_{j, \alpha} \omega_{3}^{\alpha}\left(e_{j}\right) e_{j} \wedge e_{\alpha} \wedge e_{4}-2 \sum_{j, \alpha} \omega_{4}^{\alpha}\left(e_{j}\right) e_{j} \wedge e_{3} \wedge e_{\alpha} \\
& +\sum_{i} h_{i j ; j}^{3} \mathbf{x} \wedge e_{i} \wedge e_{4}-\sum_{i} h_{i j ; j}^{4} \mathbf{x} \wedge e_{i} \wedge e_{3}
\end{aligned}
$$




$$
\begin{aligned}
& +2 \sum_{j, \alpha}\left(h_{i j}^{3} \omega_{4}^{\alpha}\left(e_{j}\right)-h_{i j}^{4} \omega_{3}^{\alpha}\left(e_{j}\right)\right) \mathbf{x} \wedge e_{i} \wedge e_{\alpha} \\
& +\sum_{j, \alpha}\left(e_{j}\left(\omega_{3}^{\alpha}\left(e_{j}\right)\right) \mathbf{x} \wedge e_{4} \wedge e_{\alpha}-e_{j}\left(\omega_{4}^{\alpha}\left(e_{j}\right)\right) \mathbf{x} \wedge e_{3} \wedge e_{\alpha}\right) \\
& -\sum_{j, \alpha}\left(\omega_{3}^{4}\left(e_{j}\right) \omega_{4}^{\alpha}\left(e_{j}\right)+\sum_{\beta} \omega_{3}^{\beta}\left(e_{j}\right) \omega_{\alpha}^{\beta}\left(e_{j}\right)\right) \mathbf{x} \wedge e_{4} \wedge e_{\alpha} \\
& +\sum_{j, \alpha}\left(\omega_{4}^{3}\left(e_{j}\right) \omega_{3}^{\alpha}\left(e_{j}\right)+\sum_{\beta} \omega_{4}^{\beta}\left(e_{j}\right) \omega_{\alpha}^{\beta}\left(e_{j}\right)\right) \mathbf{x} \wedge e_{3} \wedge e_{\alpha} .
\end{aligned}
$$

By combining (5.11), (5.12) and (5.13) and applying (2.3) of Codazzi and the minimality of $M^{2}$ in $S^{m-1}$, we obtain

$$
\begin{aligned}
\Delta^{2} \tilde{\nu}= & \left(\Delta\|\hat{h}\|^{2}+\|\hat{h}\|^{4}+4\left(K^{D}\right)^{2}\right) \tilde{\nu}+\left\{2 \Delta K^{D}\right. \\
& \left.+2 K^{D}\left(2\|\hat{h}\|^{2}+2+\sum_{\beta}\left(\left\|\omega_{3}^{\beta}\right\|^{2}+\left\|\omega_{4}^{\beta}\right\|^{2}\right)\right)\right\} \mathbf{x} \wedge e_{3} \wedge e_{4} \\
& -2 \sum_{j, r}\|\hat{h}\|_{j}^{2}\left(h_{2 j}^{r} \mathbf{x} \wedge e_{1} \wedge e_{r}-h_{1 j}^{r} \mathbf{x} \wedge e_{2} \wedge e_{r}\right) \\
& +4 \sum_{i, j} K_{j}^{D}\left(h_{i j}^{3} \mathbf{x} \wedge e_{i} \wedge e_{4}-h_{i j}^{4} \mathbf{x} \wedge e_{i} \wedge e_{3}-e_{j} \wedge e_{3} \wedge e_{4}\right) \\
& +4 K^{D} \sum_{j, \alpha}\left(h_{i j}^{3} \omega_{4}^{\alpha}\left(e_{j}\right)-h_{i j}^{4} \omega_{3}^{\alpha}\left(e_{j}\right)\right) \mathbf{x} \wedge e_{i} \wedge e_{\alpha} \\
& +4 K^{D} \sum_{j, \alpha} \omega_{3}^{\alpha}\left(e_{j}\right) e_{j} \wedge e_{4} \wedge e_{\alpha}-4 K^{D} \sum_{j, \alpha} \omega_{4}^{\alpha}\left(e_{j}\right) e_{j} \wedge e_{3} \wedge e_{\alpha} \\
& +2 \sum_{j, \alpha}\left\{K^{D}\left(\left(\nabla_{e_{j}} \omega_{3}^{\alpha}\right) e_{j}-\omega_{3}^{4}\left(e_{j}\right) \omega_{4}^{\alpha}\left(e_{j}\right)-\sum_{\beta} \omega_{3}^{\beta}\left(e_{j}\right) \omega_{\alpha}^{\beta}\left(e_{j}\right)\right)\right. \\
& \left.+2 K_{j}^{D} \omega_{3}^{\alpha}\left(e_{j}\right)\right\} \mathbf{x} \wedge e_{4} \wedge e_{\alpha} \\
& -2 \sum_{j, \alpha}\left\{K^{D}\left(\left(\nabla_{e_{j}} \omega_{4}^{\alpha}\right) e_{j}-\omega_{4}^{3}\left(e_{j}\right) \omega_{3}^{\alpha}\left(e_{j}\right)-\sum_{\beta} \omega_{4}^{\beta}\left(e_{j}\right) \omega_{\alpha}^{\beta}\left(e_{j}\right)\right)\right. \\
& \left.+2 K_{j}^{D} \omega_{4}^{\alpha}\left(e_{j}\right)\right\} \mathbf{x} \wedge e_{3} \wedge e_{\alpha} .
\end{aligned}
$$

If $\tilde{\nu}$ is mass-symmetric a 2-type, then we have $\tilde{\nu}=\tilde{\nu}_{p}+\tilde{\nu}_{q}$ with $\Delta \tilde{\nu}_{p}=$ $\lambda_{p} \tilde{\nu}_{p}, \Delta \tilde{\nu}_{q}=\lambda_{q} \tilde{\nu}_{q}, \lambda_{p} \neq \lambda_{q}$. From this we get

$$
\Delta^{2} \tilde{\nu}=\left(\lambda_{p}+\lambda_{q}\right) \Delta \tilde{\nu}-\lambda_{p} \lambda_{q} \tilde{\nu}
$$

Since $e_{j} \wedge e_{3} \wedge e_{\alpha}, e_{j} \wedge e_{\alpha} \wedge e_{4}$ appear only in $\Delta^{2} \tilde{\nu}$, and not in $\Delta \tilde{\nu}$ or in $\tilde{\nu}$, it follows from (5.14) and (5.15) that $K^{D} \omega_{3}^{\alpha}=K^{D} \omega_{4}^{\alpha}=0$. Hence, by continuity, we see that exactly one of the following two cases occurs:

(a) $K^{D}=0$ on $M^{2}$;

(b) $\omega_{3}^{\alpha}=\omega_{4}^{\alpha}=0$ and $K^{D} \neq 0$ on some non-empty open subset $U$ of $M^{2}$. 
Case (a): $K^{D}=0$ on $M^{2}$. In this case, $M^{2}$ is a minimal surface of a totally geodesic $S^{3} \subset S^{m-1}$. Thus (5.10) and (5.14) reduce to

$$
\begin{aligned}
\Delta \tilde{\nu}= & \|\hat{h}\|^{2} \tilde{\nu} \\
\Delta^{2} \tilde{\nu}= & \Delta\|\hat{h}\|^{2} \tilde{\nu}+\|\hat{h}\|^{4} \tilde{\nu} \\
& +2 \sum_{j} e_{j}\|\hat{h}\|^{2}\left\{h_{1 j}^{3} \mathbf{x} \wedge e_{2} \wedge e_{3}-h_{2 j}^{3} \mathbf{x} \wedge e_{1} \wedge e_{3}\right\} .
\end{aligned}
$$

By applying (5.15), (5.16) and (5.17), we find

$$
\|\hat{h}\|_{1}^{2} h_{11}^{3}+\|\hat{h}\|_{2}^{2} h_{12}^{3}=\|\hat{h}\|_{1}^{2} h_{12}^{3}-\|\hat{h}\|_{2}^{2} h_{11}^{3}=0 .
$$

Hence, $\|\hat{h}\|$ is constant. Thus, $\tilde{\nu}$ is of 1-type by Theorem 4.1, which is a contradiction.

Case (b) $\omega_{3}^{\alpha}=\omega_{4}^{\alpha}=0, \alpha=5, \ldots, m-1$ and $K^{D} \neq 0$ on $U$. The first normal bundle $\operatorname{Im} \hat{h}$ on $U$ is a rank 2 parallel subbundle of the normal bundle. Thus, $U$ is a minimal surface in a totally geodesic $S^{4} \subset S^{m-1}$ and (5.14) reduces to

$$
\begin{aligned}
\Delta^{2} \tilde{\nu}= & \left\{\Delta\|\hat{h}\|^{2}+\|\hat{h}\|^{4}+4\left(K^{D}\right)^{2}\right\} \tilde{\nu} \\
& +4 K^{D}\left\{1+\|\hat{h}\|^{2}\right\} \mathbf{x} \wedge e_{3} \wedge e_{4}+2 \Delta K^{D} \mathbf{x} \wedge e_{3} \wedge e_{4} \\
& +2 \sum_{j, r}\|\hat{h}\|_{j}^{2}\left(h_{1 j}^{r} \mathbf{x} \wedge e_{2} \wedge e_{r}-h_{2 j}^{r} \mathbf{x} \wedge e_{1} \wedge e_{r}\right) \\
& -4 \sum_{j} K_{j}^{D}\left\{e_{j} \wedge e_{3} \wedge e_{4}-\sum_{i} h_{i j}^{3} \mathbf{x} \wedge e_{i} \wedge e_{4}\right. \\
& \left.-\sum_{i} h_{i j}^{4} \mathbf{x} \wedge e_{3} \wedge e_{i}\right\} .
\end{aligned}
$$

Since $e_{j} \wedge e_{3} \wedge e_{4}$ appear only in $\Delta^{2} \tilde{\nu},(5.10),(5.15)$ and (5.19) imply that $K^{D}$ is constant. Therefore (5.19) becomes

$$
\begin{aligned}
\Delta^{2} \tilde{\nu}= & \left\{\Delta\|\hat{h}\|^{2}+\|\hat{h}\|^{4}+4\left(K^{D}\right)^{2}\right\} \tilde{\nu} \\
& +4 K^{D}\left\{1+\|\hat{h}\|^{2}\right\} \mathbf{x} \wedge e_{3} \wedge e_{4} \\
& +2 \sum_{j, r}\|\hat{h}\|_{j}^{2}\left(h_{1 j}^{r} \mathbf{x} \wedge e_{2} \wedge e_{r}-h_{2 j}^{r} \mathbf{x} \wedge e_{1} \wedge e_{r}\right) .
\end{aligned}
$$

It follows from (5.10), (5.15) and (5.20) that $U$ has constant curvature. Hence, a result of $[16]$ implies that $U$ is an open portion of the Veronese surface. So, by continuity, the whole $M^{2}$ is an open portion of the Veronese surface. 


\section{Spectral characterization of equilateral minimal torus}

Consider the map $\overline{\mathbf{y}}: \mathbb{E}^{2} \rightarrow \mathbb{E}^{6}$ defined by

$$
\begin{aligned}
\overline{\mathbf{y}}(s, t)= & \frac{1}{\sqrt{3}}\left(\cos \left(\frac{\sqrt{3} t+s}{\sqrt{2}}\right), \sin \left(\frac{\sqrt{3} t+s}{\sqrt{2}}\right), \cos \left(\frac{\sqrt{3} t-s}{\sqrt{2}}\right),\right. \\
& \left.\sin \left(\frac{\sqrt{3} t-s}{\sqrt{2}}\right), \cos \sqrt{2} s, \sin \sqrt{2} s\right) .
\end{aligned}
$$

Then $\overline{\mathbf{y}}$ gives rise to an isometric immersion $\mathbf{y}$ from a flat torus $\bar{T}^{2}$ into $S^{5} \subset \mathbb{E}^{6}$. The metric tensor on $\bar{T}^{2}$ induced from $(6.1)$ is $g=d s^{2}+d t^{2}$. It is easy to verify that $\Delta \overline{\mathbf{y}}=2 \overline{\mathbf{y}}$. Thus $\mathbf{y}: \bar{T}^{2} \rightarrow S^{5}$ is a minimal flat torus $\bar{T}^{2}$ in $S^{5}$, which is known as the equilateral minimal torus. This minimal torus can also be expressed as:

$$
\bar{y}(\theta, \tau)=\frac{1}{\sqrt{3}}(\cos \theta, \sin \theta, \cos \tau, \sin \tau, \cos (\theta-\tau), \sin (\theta-\tau)),
$$

with $\theta=(\sqrt{3} t+s) / \sqrt{2}$ and $\tau=(\sqrt{3} t-s) / \sqrt{2}$. However, the metric tensor on $\bar{T}^{2}$ induced from (6.2) is given by

$$
g=\frac{2}{3}\left(d \theta^{2}-d \theta d \tau+d \tau^{2}\right)
$$

instead.

Theorem 6.1. A minimal surface of $S^{m-1}$ is an open portion of the equilateral minimal torus (lying fully in a totally geodesic $S^{5} \subset S^{m-1}$ ) if and only if it has non-mass-symmetric 2-type spherical Gauss map.

Proof. First, assume that $M^{2}$ is an open portion of the equilateral minimal torus in $S^{5} \subset \mathbb{E}^{6}$ defined by (6.1). If we put

$$
\begin{aligned}
& e_{1}=\frac{\partial}{\partial s}, e_{2}=\frac{\partial}{\partial t}, e_{3}=\sqrt{2}\left(h\left(e_{1}, e_{1}\right)+\bar{y}\right), \\
& e_{4}=\sqrt{2} h\left(e_{1}, e_{2}\right), \quad e_{5}=D_{\partial / \partial s} e_{3}, e_{6}=\bar{y},
\end{aligned}
$$

then $e_{3}, e_{4}, e_{5}, e_{6}$ are orthonormal normal vector fields. A direct computation gives

$$
\begin{aligned}
& h_{11}^{3}=-h_{22}^{3}=h_{12}^{4}=\frac{1}{\sqrt{2}}, h_{12}^{3}=h_{11}^{4}=h_{22}^{4}=h_{i j}^{\alpha}=0, \\
& \omega_{1}^{2}=\omega_{3}^{4}=0, \quad \omega_{3}^{5}=\omega^{1}, \quad \omega_{4}^{5}=-\omega^{2}, \alpha=5,6 ; i, j=1,2 .
\end{aligned}
$$

It follows from $(5.10),(5.15)$ and (6.4) that the spherical Gauss map satisfies

$$
\begin{aligned}
& \Delta \tilde{\nu}=2 \tilde{\nu}-2 \bar{y} \wedge e_{3} \wedge e_{4}, \\
& \Delta^{2} \tilde{\nu}=8 \tilde{\nu}-16 \bar{y} \wedge e_{3} \wedge e_{4}-4 e_{1} \wedge e_{4} \wedge e_{5}-4 e_{2} \wedge e_{3} \wedge e_{5} .
\end{aligned}
$$

Put $c=\frac{1}{4}\left(\tilde{\nu}+\bar{y} \wedge e_{3} \wedge e_{4}-e_{1} \wedge e_{4} \wedge e_{5}-e_{2} \wedge e_{3} \wedge e_{5}\right)$. Then we see that $c$ is a constant vector by differentiating $c$ and applying (2.1), (3.3), and (6.4). If 
we put

$$
\tilde{\nu}_{1}=\tilde{\nu}+\frac{1}{3} \bar{y} \wedge e_{3} \wedge e_{4}-\frac{4}{3} c, \quad \tilde{\nu}_{2}=\frac{1}{3}\left(c-\bar{y} \wedge e_{3} \wedge e_{4}\right)
$$

a straight-forward long computation yields $\tilde{\nu}=c+\tilde{\nu}_{1}+\tilde{\nu}_{2}$ with $\Delta \tilde{\nu}_{1}=2 \tilde{\nu}_{1}$ and $\Delta \tilde{\nu}_{2}=8 \tilde{\nu}_{2}$. Thus, $\tilde{\nu}$ is non-mass-symmetric 2-type.

Conversely, assume $M^{2}$ has non-mass-symmetric 2-type spherical Gauss map. If we choose $e_{1}, e_{2}, e_{3}, \ldots, e_{m}$ as in the proof of Theorem 5.1 and use the same convention on the range of indices, we obtain (5.9), (5.11) and (5.14).

From (3.3) and (5.10), (5.14), and a very long computation, we obtain

$$
\begin{aligned}
e_{k} \tilde{\nu}= & \sum_{r}\left(h_{2 k}^{r} \mathbf{x} \wedge e_{1} \wedge e_{r}-h_{1 k}^{r} \mathbf{x} \wedge e_{2} \wedge e_{r}\right) \\
e_{k}(\Delta \tilde{\nu})= & \|\hat{h}\|_{k}^{2} \tilde{\nu}+\|\hat{h}\|^{2} \sum_{r}\left(h_{2 k}^{r} \mathbf{x} \wedge e_{1} \wedge e_{r}-h_{1 k}^{r} \mathbf{x} \wedge e_{2} \wedge e_{r}\right) \\
& +2 K_{k}^{D} \mathbf{x} \wedge e_{3} \wedge e_{4}+2 K^{D}\left\{e_{k} \wedge e_{3} \wedge e_{4}-\sum_{j} h_{j k}^{3} \mathbf{x} \wedge e_{j} \wedge e_{4}\right. \\
& +\sum_{j} h_{j k}^{4} \mathbf{x} \wedge e_{j} \wedge e_{3}-\sum_{\alpha} \omega_{3}^{\alpha}\left(e_{k}\right) \mathbf{x} \wedge e_{4} \wedge e_{\alpha} \\
& \left.+\sum_{\alpha} \omega_{4}^{\alpha}\left(e_{k}\right) \mathbf{x} \wedge e_{3} \wedge e_{\alpha}\right\}
\end{aligned}
$$

and

(6.8)

$$
\begin{aligned}
e_{k}\left(\Delta^{2} \tilde{\nu}\right)= & \left\{e_{k}\left(\Delta\|\hat{h}\|^{2}\right)+e_{k}\left(\|\hat{h}\|^{4}\right)+8 K^{D} K_{k}^{D}\right\} \tilde{\nu} \\
& +2\left\{e_{k}\left(\Delta K^{D}\right)+2 K_{k}^{D}+2 K_{k}^{D}\|\hat{h}\|^{2}+K_{k}^{D} \sum_{\beta}\left(\left\|\omega_{3}^{\beta}\right\|^{2}+\left\|\omega_{4}^{\beta}\right\|^{2}\right)\right. \\
& \left.+2 K^{D}\|\hat{h}\|_{k}^{2}+K^{D} \sum_{\beta}\left(\left\|\omega_{3}^{\beta}\right\|_{k}^{2}+\left\|\omega_{4}^{\beta}\right\|_{k}^{2}\right)\right\} \mathbf{x} \wedge e_{3} \wedge e_{4} \\
& -2 \sum_{j, r}\left(\|\hat{h}\|_{j k}^{2} h_{2 j}^{r}+\|\hat{h}\|_{j}^{2} e_{k}\left(h_{2 j}^{r}\right)\right) \mathbf{x} \wedge e_{1} \wedge e_{r} \\
& +2 \sum_{j, r}\left(\|\hat{h}\|_{j k}^{2} h_{1 j}^{r}+\|\hat{h}\|_{j}^{2} e_{k}\left(h_{1 j}^{r}\right)\right) \mathbf{x} \wedge e_{2} \wedge e_{r} \\
& +4 \sum_{j} K_{j k}^{D}\left\{\sum_{i} h_{i j}^{3} \mathbf{x} \wedge e_{i} \wedge e_{4}-\sum_{i} h_{i j}^{4} \mathbf{x} \wedge e_{i} \wedge e_{3}\right. \\
& \left.-\sum_{\alpha} \omega_{3}^{\alpha}\left(e_{j}\right) \mathbf{x} \wedge e_{\alpha} \wedge e_{4}-\sum_{\alpha} \omega_{4}^{\alpha}\left(e_{j}\right) \mathbf{x} \wedge e_{3} \wedge e_{\alpha}-e_{j} \wedge e_{3} \wedge e_{4}\right\} \\
& +4 \sum_{j} K_{j}^{D}\left\{\sum_{i} e_{k}\left(h_{i j}^{3}\right) \mathbf{x} \wedge e_{i} \wedge e_{4}-\sum_{i} e_{k}\left(h_{i j}^{4}\right) \mathbf{x} \wedge e_{i} \wedge e_{3}\right.
\end{aligned}
$$




$$
\begin{aligned}
& \left.+\sum_{\alpha} e_{k}\left(\omega_{3}^{\alpha}\left(e_{j}\right)\right) \mathbf{x} \wedge e_{4} \wedge e_{\alpha}-\sum_{\alpha} e_{k}\left(\omega_{4}^{\alpha}\left(e_{j}\right)\right) \mathbf{x} \wedge e_{3} \wedge e_{\alpha}\right\} \\
& +2 K_{k}^{D} \sum_{j, \alpha}\left\{2\left(h_{i j}^{3} \omega_{4}^{\alpha}\left(e_{j}\right)-h_{i j}^{4} \omega_{3}^{\alpha}\left(e_{j}\right)\right) \mathbf{x} \wedge e_{i} \wedge e_{\alpha}\right. \\
& +\left[\left(\nabla_{e_{j}} \omega_{3}^{\alpha}\right) e_{j}-\omega_{3}^{4}\left(e_{j}\right) \omega_{4}^{\alpha}\left(e_{j}\right)-\sum_{\beta} \omega_{3}^{\beta}\left(e_{j}\right) \omega_{\alpha}^{\beta}\left(e_{j}\right)\right] \mathbf{x} \wedge e_{4} \wedge e_{\alpha} \\
& -\left[\left(\nabla_{e_{j}} \omega_{4}^{\alpha}\right) e_{j}-\omega_{4}^{3}\left(e_{j}\right) \omega_{3}^{\alpha}\left(e_{j}\right)-\sum_{\beta} \omega_{4}^{\beta}\left(e_{j}\right) \omega_{\alpha}^{\beta}\left(e_{j}\right)\right] \mathrm{x} \wedge e_{3} \wedge e_{\alpha} \\
& \left.+2 \omega_{3}^{\alpha}\left(e_{j}\right) e_{j} \wedge e_{4} \wedge e_{\alpha}-2 \omega_{4}^{\alpha}\left(e_{j}\right) e_{j} \wedge e_{3} \wedge e_{\alpha}\right\} \\
& +2 K^{D} \sum_{j, \alpha}\left\{2 e_{k}\left(h_{i j}^{3} \omega_{4}^{\alpha}\left(e_{j}\right)-h_{i j}^{4} \omega_{3}^{\alpha}\left(e_{j}\right)\right) \times \wedge e_{i} \wedge e_{\alpha}\right. \\
& \left.+e_{k}\left[\left(\nabla_{e_{j}} \omega_{3}^{\alpha}\right) e_{j}-\omega_{3}^{4}\left(e_{j}\right) \omega_{4}^{\alpha}\left(e_{j}\right)-\sum_{\beta} \omega_{3}^{\beta}\left(e_{j}\right) \omega_{\alpha}^{\beta}\left(e_{j}\right)\right] \mathbf{x} \wedge e_{4} \wedge e_{\alpha}\right\} \\
& -2 K^{D} \sum_{j, \alpha} e_{k}\left[\left(\nabla_{e_{j}} \omega_{4}^{\alpha}\right) e_{j}-\omega_{4}^{3}\left(e_{j}\right) \omega_{3}^{\alpha}\left(e_{j}\right)-\sum_{\beta} \omega_{4}^{\beta}\left(e_{j}\right) \omega_{\alpha}^{\beta}\left(e_{j}\right)\right] \mathbf{x} \wedge e_{3} \wedge e_{\alpha} \\
& +4 K^{D} \sum_{j, \alpha} e_{k}\left(\omega_{3}^{\alpha}\left(e_{j}\right)\right) e_{j} \wedge e_{4} \wedge e_{\alpha}-4 K^{D} \sum_{j, \alpha} e_{k}\left(\omega_{4}^{\alpha}\left(e_{j}\right)\right) e_{j} \wedge e_{3} \wedge e_{\alpha} \\
& +\left(\Delta\|\hat{h}\|^{2}+\|\hat{h}\|^{4}+4\left(K^{D}\right)^{2}\right) \sum_{r}\left(h_{2 k}^{r} \mathbf{x} \wedge e_{1} \wedge e_{r}-h_{1 k}^{r} \mathbf{x} \wedge e_{2} \wedge e_{r}\right) \\
& +2\left\{\Delta K^{D}+K^{D}\left(2+2\|\hat{h}\|^{2}+\sum_{\beta}\left(\left\|\omega_{3}^{\beta}\right\|^{2}+\left\|\omega_{4}^{\beta}\right\|^{2}\right)\right)\right\} \\
& \times\left\{e_{k} \wedge e_{3} \wedge e_{4}-\sum_{j} h_{j k}^{3} \mathbf{x} \wedge e_{j} \wedge e_{4}+\sum_{j} h_{j k}^{4} \mathbf{x} \wedge e_{j} \wedge e_{3}\right. \\
& \left.-\sum_{\alpha} \omega_{3}^{\alpha}\left(e_{k}\right) \mathbf{x} \wedge e_{4} \wedge e_{\alpha}+\sum_{\alpha} \omega_{4}^{\alpha}\left(e_{k}\right) \mathbf{x} \wedge e_{3} \wedge e_{\alpha}\right\} \\
& -2 \sum_{j, r}\|\hat{h}\|_{j}^{2} h_{2 j}^{r}\left\{e_{k} \wedge e_{1} \wedge e_{r}+\omega_{1}^{2}\left(e_{k}\right) \mathbf{x} \wedge e_{2} \wedge e_{r}+\sum_{s} h_{1 k}^{s} \mathbf{x} \wedge e_{s} \wedge e_{r}\right. \\
& \left.-h_{2 k}^{r} \tilde{\nu}+\sum_{s} \omega_{r}^{s}\left(e_{k}\right) \mathbf{x} \wedge e_{1} \wedge e_{s}-\sum_{\alpha} \omega_{r}^{\alpha}\left(e_{k}\right) \mathbf{x} \wedge e_{1} \wedge e_{\alpha}\right\} \\
& +2 \sum_{j, r}\|\hat{h}\|_{j}^{2} h_{1 j}^{r}\left\{e_{k} \wedge e_{2} \wedge e_{r}-\omega_{1}^{2}\left(e_{k}\right) \mathbf{x} \wedge e_{1} \wedge e_{r}+\sum_{s} h_{2 k}^{s} \mathbf{x} \wedge e_{s} \wedge e_{r}\right. \\
& \left.+h_{1 k}^{r} \tilde{\nu}+\sum_{s} \omega_{r}^{s}\left(e_{k}\right) \mathbf{x} \wedge e_{2} \wedge e_{s}+\sum_{\alpha} \omega_{r}^{\alpha}\left(e_{k}\right) \mathbf{x} \wedge e_{2} \wedge e_{\alpha}\right\} \\
& +4 \sum_{i, j} K_{j}^{D} h_{i j}^{3}\left\{e_{k} \wedge e_{i} \wedge e_{4}+\sum_{\ell} \omega_{i}^{\ell}\left(e_{k}\right) \mathbf{x} \wedge e_{\ell} \wedge e_{4}+h_{i k}^{3} \mathbf{x} \wedge e_{3} \wedge e_{4}\right. \\
& \left.-\sum_{\ell} h_{k \ell}^{4} \mathbf{x} \wedge e_{i} \wedge e_{\ell}-\omega_{3}^{4}\left(e_{k}\right) \mathbf{x} \wedge e_{i} \wedge e_{3}+\sum_{\alpha} \omega_{4}^{\alpha}\left(e_{k}\right) \mathbf{x} \wedge e_{i} \wedge e_{\alpha}\right\}
\end{aligned}
$$


$-4 \sum_{i, j} K_{j}^{D} h_{i j}^{4}\left\{e_{k} \wedge e_{i} \wedge e_{3}+h_{k \ell}^{3} \mathbf{x} \wedge e_{\ell} \wedge e_{i}+\omega_{3}^{4}\left(e_{k}\right) \mathbf{x} \wedge e_{i} \wedge e_{4}\right.$

$\left.+\sum_{\alpha} \omega_{3}^{\alpha}\left(e_{k}\right) \mathbf{x} \wedge e_{i} \wedge e_{\alpha}+\sum_{\ell} \omega_{i}^{\ell}\left(e_{k}\right) \mathbf{x} \wedge e_{\ell} \wedge e_{3}-h_{i k}^{4} \mathbf{x} \wedge e_{3} \wedge e_{4}\right\}$

$-4 \sum_{j} K_{j}^{D}\left\{\sum_{i} \omega_{j}^{i}\left(e_{k}\right) e_{i} \wedge e_{3} \wedge e_{4}-\sum_{i} h_{i k}^{3} e_{j} \wedge e_{i} \wedge e_{4}\right.$

$\left.-\sum_{\alpha} \omega_{3}^{\alpha}\left(e_{k}\right) e_{j} \wedge e_{4} \wedge e_{\alpha}+\sum_{i} h_{i k}^{4} e_{j} \wedge e_{i} \wedge e_{3}+\sum_{\alpha} \omega_{4}^{\alpha}\left(e_{k}\right) e_{j} \wedge e_{3} \wedge e_{\alpha}\right\}$

$+2 \sum_{j, \alpha}\left\{K^{D}\left[\left(\nabla_{e_{j}} \omega_{3}^{\alpha}\right) e_{j}-\omega_{3}^{4}\left(e_{j}\right) \omega_{4}^{\alpha}\left(e_{j}\right)-\sum_{\gamma} \omega_{3}^{\gamma}\left(e_{j}\right) \omega_{\alpha}^{\gamma}\left(e_{j}\right)\right]\right.$

$\left.+2 K_{j}^{D} \omega_{3}^{\alpha}\left(e_{j}\right)\right\}\left\{e_{k} \wedge e_{4} \wedge e_{\alpha}-\sum_{i} h_{i k}^{4} \mathbf{x} \wedge e_{i} \wedge e_{\alpha}-\omega_{3}^{4}\left(e_{k}\right) \mathbf{x} \wedge e_{3} \wedge e_{\alpha}\right.$

$\left.+\sum_{\beta} \omega_{4}^{\beta}\left(e_{k}\right) \mathbf{x} \wedge e_{\beta} \wedge e_{\alpha}+\omega_{3}^{\alpha}\left(e_{k}\right) \mathbf{x} \wedge e_{3} \wedge e_{4}+\sum_{\beta} \omega_{\alpha}^{\beta}\left(e_{k}\right) \mathbf{x} \wedge e_{4} \wedge e_{\beta}\right\}$

$-2 \sum_{j, \alpha}\left\{K^{D}\left[\left(\nabla_{e_{j}} \omega_{4}^{\alpha}\right) e_{j}-\omega_{4}^{3}\left(e_{j}\right) \omega_{3}^{\alpha}\left(e_{j}\right)-\sum_{\gamma} \omega_{4}^{\gamma}\left(e_{j}\right) \omega_{\alpha}^{\gamma}\left(e_{j}\right)\right]\right.$

$\left.+2 K_{j}^{D} \omega_{4}^{\alpha}\left(e_{j}\right)\right\}\left\{e_{k} \wedge e_{3} \wedge e_{\alpha}-\sum_{i} h_{i k}^{3} \mathbf{x} \wedge e_{i} \wedge e_{\alpha}\right.$

$+\omega_{3}^{4}\left(e_{k}\right) \mathbf{x} \wedge e_{4} \wedge e_{\alpha}-\omega_{4}^{\alpha}\left(e_{k}\right) \mathbf{x} \wedge e_{3} \wedge e_{4}+\sum_{\beta} \omega_{3}^{\beta}\left(e_{k}\right) \mathbf{x} \wedge e_{\beta} \wedge e_{\alpha}$

$\left.+\sum_{\beta} \omega_{\alpha}^{\beta}\left(e_{k}\right) \mathbf{x} \wedge e_{3} \wedge e_{\beta}\right\}+4 K^{D} \sum_{j, \alpha}\left(h_{i j}^{3} \omega_{4}^{\alpha}\left(e_{j}\right)-h_{i j}^{4} \omega_{3}^{\alpha}\left(e_{j}\right)\right)$

$\times\left\{e_{k} \wedge e_{i} \wedge e_{\alpha}+\sum_{\ell} \omega_{i}^{\ell}\left(e_{k}\right) \mathbf{x} \wedge e_{\ell} \wedge e_{\alpha}+\sum_{r} h_{i k}^{r} \mathbf{x} \wedge e_{r} \wedge e_{\alpha}\right.$

$\left.-\omega_{3}^{\alpha}\left(e_{k}\right) \mathbf{x} \wedge e_{i} \wedge e_{3}-\omega_{4}^{\alpha}\left(e_{k}\right) \mathbf{x} \wedge e_{i} \wedge e_{4}+\sum_{\beta} \omega_{\alpha}^{\beta}\left(e_{k}\right) \mathbf{x} \wedge e_{i} \wedge e_{\beta}\right\}$

$+4 K^{D} \sum_{j, \alpha} \omega_{3}^{\alpha}\left(e_{j}\right)\left\{\sum_{i} \omega_{j}^{i}\left(e_{k}\right) e_{i} \wedge e_{4} \wedge e_{\alpha}+h_{j k}^{3} e_{3} \wedge e_{4} \wedge e_{\alpha}\right.$

$+\omega_{3}^{\alpha}\left(e_{k}\right) e_{j} \wedge e_{3} \wedge e_{4}+\sum_{\beta} \omega_{\alpha}^{\beta}\left(e_{k}\right) e_{j} \wedge e_{4} \wedge e_{\beta}-\sum_{i} h_{i k}^{4} e_{j} \wedge e_{i} \wedge e_{\alpha}$

$\left.-\omega_{3}^{4}\left(e_{k}\right) e_{j} \wedge e_{3} \wedge e_{\alpha}-\sum_{\beta} \omega_{4}^{\beta}\left(e_{k}\right) e_{j} \wedge e_{\alpha} \wedge e_{\beta}\right\}$

$-4 K^{D} \sum_{j, \alpha} \omega_{4}^{\alpha}\left(e_{j}\right)\left\{\sum_{i} \omega_{j}^{i}\left(e_{k}\right) e_{i} \wedge e_{3} \wedge e_{\alpha}-h_{j k}^{4} e_{3} \wedge e_{4} \wedge e_{\alpha}\right.$

$-\sum_{i} h_{i k}^{3} e_{j} \wedge e_{i} \wedge e_{\alpha}+\omega_{3}^{4}\left(e_{k}\right) e_{j} \wedge e_{4} \wedge e_{\alpha}-\sum_{\beta} \omega_{3}^{\beta}\left(e_{k}\right) e_{j} \wedge e_{\alpha} \wedge e_{\beta}$ 


$$
\left.-\omega_{4}^{\alpha}\left(e_{k}\right) e_{j} \wedge e_{3} \wedge e_{4}+\sum_{\beta} \omega_{\alpha}^{\beta}\left(e_{k}\right) e_{j} \wedge e_{3} \wedge e_{\beta}\right\}
$$

where $K_{j k}^{D}=e_{k} e_{j} K^{D}$ and $\|\hat{h}\|_{j k}^{2}=e_{k} e_{j}\|\hat{h}\|^{2}$. Since $M^{2}$ has non-masssymmetric 2-type spherical Gauss map, we have $\tilde{\nu}=c+\tilde{\nu}_{1}+\tilde{\nu}_{2}$ with $\Delta \tilde{\nu}_{1}=\lambda_{p} \tilde{\nu}_{1}$, $\Delta \tilde{\nu}_{2}=\lambda_{q} \tilde{\nu}_{2}, \lambda_{p} \neq \lambda_{q}$ and nonzero vector $c$. Hence, we get

$$
e_{k}\left(\Delta^{2} \tilde{\nu}\right)-\left(\lambda_{p}+\lambda_{q}\right) e_{k}(\Delta \tilde{\nu})+\lambda_{p} \lambda_{q} e_{k} \tilde{\nu}=0, \quad k=1,2 .
$$

If $K^{D}$ vanishes identically on $M^{2}$, then $M^{2}$ lies in a totally geodesic $S^{3} \subset$ $S^{m-1}$. So, we may assume $m=4$. Hence, $(6.7)$ and (6.8) reduce to

$$
\begin{aligned}
e_{k} \tilde{\nu}= & \sum_{r}\left(h_{2 k}^{r} \mathbf{x} \wedge e_{1} \wedge e_{r}-h_{1 k}^{r} \mathbf{x} \wedge e_{2} \wedge e_{r}\right) \\
e_{k}(\Delta \tilde{\nu})= & \|\hat{h}\|_{k}^{2} \tilde{\nu}+\|\hat{h}\|^{2}\left(h_{2 k}^{3} \mathbf{x} \wedge e_{1} \wedge e_{3}-h_{1 k}^{3} \mathbf{x} \wedge e_{2} \wedge e_{3}\right), \\
e_{k}\left(\Delta^{2} \tilde{\nu}\right)= & \left\{e_{k}\left(\Delta\|\hat{h}\|^{2}\right)+\|\hat{h}\|_{k}^{4}\right\} \tilde{\nu} \\
& -2 \sum_{j}\left(\|\hat{h}\|_{j k}^{2} h_{2 j}^{3}+\|\hat{h}\|_{j}^{2} e_{k}\left(h_{2 j}^{3}\right)\right) \mathbf{x} \wedge e_{1} \wedge e_{3} \\
& +2 \sum_{j}\left(\|\hat{h}\|_{j k}^{2} h_{1 j}^{3}+\|\hat{h}\|_{j}^{2} e_{k}\left(h_{1 j}^{3}\right)\right) \mathbf{x} \wedge e_{2} \wedge e_{3} \\
& +\left(\Delta\|\hat{h}\|^{2}+\|\hat{h}\|^{4}\right)\left(h_{2 k}^{3} \mathbf{x} \wedge e_{1} \wedge e_{3}-h_{1 k}^{3} \mathbf{x} \wedge e_{2} \wedge e_{3}\right) \\
& +2 \sum_{j}\|\hat{h}\|_{j}^{2} h_{2 j}^{3}\left\{\delta_{2 k} e_{1} \wedge e_{2} \wedge e_{3}-\omega_{1}^{2}\left(e_{k}\right) \mathbf{x} \wedge e_{2} \wedge e_{3}+h_{2 k}^{3} \tilde{\nu}\right\} \\
& +2 \sum_{j}\|\hat{h}\|_{j}^{2} h_{1 j}^{3}\left\{\delta_{1 k} e_{1} \wedge e_{2} \wedge e_{3}-\omega_{1}^{2}\left(e_{k}\right) \mathbf{x} \wedge e_{1} \wedge e_{3}+h_{1 k}^{3} \tilde{\nu}\right\} .
\end{aligned}
$$

We see from (6.10) that $e_{1} \wedge e_{2} \wedge e_{3}$ appears in $e_{k}\left(\Delta^{2} \tilde{\nu}\right)$, not in $e_{k}(\Delta \tilde{\nu})$ or in $e_{k} \tilde{\nu}$. Thus, (6.9) and (6.10) imply that $\|\hat{h}\|$ is constant. So, according to Theorem $4.1, \tilde{\nu}$ is mass-symmetric 1-type which is a contradiction. Since $M^{2}$ is analytic, those imply $K^{D} \neq 0$ on an open dense subset $U$ of $M^{2}$.

From $K^{D} \neq 0$ on $U$, we may choose $e_{1}, e_{2}, e_{3}, \ldots, e_{m-1}$ on $U$ such that $A_{e_{3}}, A_{e_{4}} \neq 0, A_{e_{5}}=\cdots=A_{e_{m-1}}=h_{12}^{3}=0, D_{e_{1}} e_{3}$ lies in the linear subspace spanned by $e_{4}, e_{5}$ and $D_{e_{2}} e_{3}$ lies in the linear subspace spanned by $e_{4}, e_{5}, e_{6}$. From these we have

$$
\begin{gathered}
\omega_{3}^{6}\left(e_{1}\right)=\omega_{3}^{7}=\cdots=\omega_{3}^{m-1}=h_{12}^{3}=h_{j k}^{\alpha}=0, \\
\alpha=5, \ldots, m-1, \\
K^{D}=-2 h_{11}^{3} h_{12}^{4} \neq 0, \quad h_{11}^{3}=-h_{22}^{3} \neq 0
\end{gathered}
$$

on the dense open subset $U$. 
We see from (6.7) and (6.8) that $e_{1} \wedge e_{2} \wedge e_{\alpha}$ appears in $e_{k}\left(\Delta^{2} \tilde{\nu}\right)$, not in $e_{k}(\Delta \tilde{\nu})$ or in $e_{k} \tilde{\nu}$. Thus, (6.8) and (6.9) yield

$$
\begin{aligned}
& \sum_{j}\left(h_{i j}^{3} \omega_{4}^{\alpha}\left(e_{j}\right)-h_{i j}^{4} \omega_{3}^{\alpha}\left(e_{j}\right)\right) e_{k} \wedge e_{i} \wedge e_{\alpha} \\
= & \sum_{i, j} \omega_{3}^{\alpha}\left(e_{j}\right) h_{i k}^{4} e_{j} \wedge e_{i} \wedge e_{\alpha}-\sum_{i, j} \omega_{4}^{\alpha}\left(e_{j}\right) h_{i k}^{3} e_{j} \wedge e_{i} \wedge e_{\alpha}
\end{aligned}
$$

for $k=1,2 ; \alpha=5, \ldots, m-1$.

In view of (6.11), we find from (6.13) that

$$
\begin{aligned}
& h_{11}^{4} \omega_{3}^{\alpha}\left(e_{2}\right)-h_{12}^{4} \omega_{3}^{\alpha}\left(e_{1}\right)=h_{11}^{3} \omega_{4}^{\alpha}\left(e_{2}\right), \\
& h_{11}^{4} \omega_{3}^{\alpha}\left(e_{1}\right)+h_{12}^{4} \omega_{3}^{\alpha}\left(e_{2}\right)=h_{11}^{3} \omega_{4}^{\alpha}\left(e_{1}\right)
\end{aligned}
$$

for $\alpha=5, \ldots, m-1$. Combining these with $\omega_{3}^{6}\left(e_{1}\right)=0$ gives

$$
h_{11}^{4} \omega_{3}^{6}\left(e_{2}\right)=h_{11}^{3} \omega_{4}^{6}\left(e_{2}\right), h_{12}^{4} \omega_{3}^{6}\left(e_{2}\right)=h_{11}^{3} \omega_{4}^{6}\left(e_{1}\right),
$$

which implies that

$$
h_{11}^{4} \omega_{4}^{6}\left(e_{1}\right)=h_{12}^{4} \omega_{4}^{6}\left(e_{2}\right) .
$$

On the other hand, we see from (6.7) and (6.8) that $e_{3} \wedge e_{4} \wedge e_{\alpha}$ appears only in $e_{k}\left(\Delta^{2} \tilde{\nu}\right)$, not in $e_{k}(\Delta \tilde{\nu})$ or in $e_{k} \tilde{\nu}$. Thus, (6.8) and (6.9) give

$$
\sum_{j} \omega_{3}^{\alpha}\left(e_{j}\right) h_{j k}^{3}+\sum_{j} \omega_{4}^{\alpha}\left(e_{j}\right) h_{j k}^{4}=0
$$

for $k=1,2$ and $\alpha=5, \ldots, m-1$. Hence, in view of $(6.11)$, we find

$$
\begin{aligned}
& \omega_{3}^{\alpha}\left(e_{1}\right) h_{11}^{3}+\omega_{4}^{\alpha}\left(e_{1}\right) h_{11}^{4}+\omega_{4}^{\alpha}\left(e_{2}\right) h_{12}^{4}=0, \\
& \omega_{3}^{\alpha}\left(e_{2}\right) h_{11}^{3}-\omega_{4}^{\alpha}\left(e_{1}\right) h_{12}^{4}+\omega_{4}^{\alpha}\left(e_{2}\right) h_{11}^{4}=0
\end{aligned}
$$

for $\alpha=5, \ldots, m-1$. In particular, we have $\omega_{4}^{6}\left(e_{1}\right) h_{11}^{4}+\omega_{4}^{6}\left(e_{2}\right) h_{12}^{4}=0$. Combining this with (6.11) and (6.17) gives

$$
h_{11}^{4} \omega_{4}^{6}\left(e_{1}\right)=0, \quad \omega_{3}^{6}\left(e_{1}\right)=\omega_{4}^{6}\left(e_{2}\right)=0 .
$$

By substituting the second equation of (6.21) into (6.16) and (6.20), we obtain

$$
h_{11}^{4} \omega_{3}^{6}\left(e_{2}\right)=0, \omega_{3}^{6}\left(e_{2}\right) h_{11}^{3}=\omega_{4}^{6}\left(e_{1}\right) h_{12}^{4} .
$$

Also we obtain from (6.11), (6.19) and (6.20) that

$$
\omega_{4}^{\alpha}\left(e_{1}\right) h_{11}^{4}+\omega_{4}^{\alpha}\left(e_{2}\right) h_{12}^{4}=\omega_{4}^{\alpha}\left(e_{1}\right) h_{12}^{4}-\omega_{4}^{\alpha}\left(e_{2}\right) h_{11}^{4}=0
$$

for $\alpha=7, \ldots, m-1$. Combining this with (6.11) gives

$$
\omega_{3}^{7}=\cdots=\omega_{3}^{m-1}=\omega_{4}^{7}=\cdots=\omega_{4}^{m-1}=0 .
$$

We see from (6.7) and (6.8) that $e_{j} \wedge e_{5} \wedge e_{6}$ appears in $e_{k}\left(\Delta^{2} \tilde{\nu}\right)$, not in $e_{k}(\Delta \tilde{\nu})$ or in $e_{k} \tilde{\nu}=0$. Thus, we obtain from $(6.8),(6.9),(6.11)$ and (6.21) that

$$
\omega_{4}^{5}\left(e_{1}\right) \omega_{3}^{6}\left(e_{2}\right)=\omega_{4}^{6}\left(e_{1}\right) \omega_{3}^{5}\left(e_{2}\right) \text {. }
$$

Now, we divide the proof into two cases: 
Case (i): $h_{11}^{4} \neq 0$. From (6.21)-(6.23) we obtain $\omega_{3}^{\alpha}=\omega_{4}^{\alpha}=0$ for $\alpha=$ $6, \ldots, m-1$. Also, from $(6.14),(6.15),(6.19)$ and $(6.20)$, we have

$$
\begin{aligned}
& h_{11}^{3} \omega_{4}^{5}\left(e_{2}\right)-h_{11}^{4} \omega_{3}^{5}\left(e_{2}\right)+h_{12}^{4} \omega_{3}^{5}\left(e_{1}\right)=0 \\
& h_{11}^{3} \omega_{4}^{5}\left(e_{1}\right)-h_{11}^{4} \omega_{3}^{5}\left(e_{1}\right)-h_{12}^{4} \omega_{3}^{5}\left(e_{2}\right)=0 \\
& h_{11}^{3} \omega_{3}^{5}\left(e_{1}\right)+h_{11}^{4} \omega_{4}^{5}\left(e_{1}\right)+h_{12}^{4} \omega_{4}^{5}\left(e_{2}\right)=0 \\
& h_{11}^{3} \omega_{3}^{5}\left(e_{2}\right)+h_{11}^{4} \omega_{4}^{5}\left(e_{2}\right)-h_{12}^{4} \omega_{4}^{5}\left(e_{1}\right)=0 .
\end{aligned}
$$

After solving this linear system we obtain $\omega_{3}^{5}=\omega_{4}^{5}=0$. Consequently, we have

$$
\omega_{3}^{\alpha}=\omega_{4}^{\alpha}=0, \alpha=5, \ldots, m-1
$$

Since the first normal bundle, $\operatorname{Im} \hat{h}$, is spanned by $e_{3}, e_{4}$ on $U,(6.26)$ implies that the first normal bundle is a parallel subbundle of the normal bundle. Hence, the reduction theorem of Erbarcher implies that $M^{2}$ lies in a totally geodesic $S^{4} \subset S^{m-1}$. So, without loss of generality, we may assume that $m=5$.

Now, by comparing the coefficients of $e_{1} \wedge e_{2} \wedge e_{3}$ and of $e_{1} \wedge e_{2} \wedge e_{4}$, we obtain from $(6.7),(6.8)$ and $(6.9)$ that

$$
\begin{aligned}
\|\hat{h}\|_{1}^{2} h_{11}^{3}= & 4 K_{1}^{D} h_{12}^{4}-4 K_{2}^{D} h_{11}^{4},\|\hat{h}\|_{2}^{2} h_{11}^{3}=4 K_{1}^{D} h_{11}^{4}+4 K_{2}^{D} h_{12}^{4}, \\
& \|\hat{h}\|_{1}^{2} h_{11}^{4}+\|\hat{h}\|_{2}^{2} h_{12}^{4}=4 K_{2}^{D} h_{11}^{3},\|\hat{h}\|_{1}^{2} h_{12}^{4}-\|\hat{h}\|_{2}^{2} h_{11}^{4}=4 K_{1}^{D} h_{11}^{3} .
\end{aligned}
$$

After solving this system we obtain $K_{1}^{D}=K_{2}^{D}=0$. Thus, the normal curvature $K^{D}$ is a nonzero constant. Therefore, equations (6.7) and (6.8) reduce to

$$
\begin{aligned}
e_{k} \tilde{\nu}= & \sum_{r}\left(h_{2 k}^{r} \mathbf{x} \wedge e_{1} \wedge e_{r}-h_{1 k}^{r} \mathbf{x} \wedge e_{2} \wedge e_{r}\right), \\
e_{k}(\Delta \tilde{\nu})= & \|\hat{h}\|_{k}^{2} \tilde{\nu}+\|\hat{h}\|^{2} \sum_{r}\left(h_{2 k}^{r} \mathbf{x} \wedge e_{1} \wedge e_{r}-h_{1 k}^{r} \mathbf{x} \wedge e_{2} \wedge e_{r}\right) \\
& +2 K^{D}\left\{e_{k} \wedge e_{3} \wedge e_{4}-\sum_{j} h_{j k}^{3} \mathbf{x} \wedge e_{j} \wedge e_{4}+\sum_{j} h_{j k}^{4} \mathbf{x} \wedge e_{j} \wedge e_{3}\right\}, \\
e_{k}\left(\Delta^{2} \tilde{\nu}\right)= & \left\{e_{k}\left(\Delta\|\hat{h}\|^{2}\right)+\|\hat{h}\|_{k}^{4}+2 \sum_{i, j, r}\|\hat{h}\|_{j}^{2} h_{i j}^{r} h_{i k}^{r}\right\} \tilde{\nu} \\
& -2 \sum_{j, r}\left(\|\hat{h}\|_{j k}^{2} h_{2 j}^{r}+\|\hat{h}\|_{j}^{2} e_{k}\left(h_{2 j}^{r}\right)\right) \mathbf{x} \wedge e_{1} \wedge e_{r} \\
& +2 \sum_{j, r}\left(\|\hat{h}\|_{j k}^{2} h_{1 j}^{r}+\|\hat{h}\|_{j}^{2} e_{k}\left(h_{1 j}^{r}\right)\right) \mathbf{x} \wedge e_{2} \wedge e_{r} \\
& +\left(\Delta\|\hat{h}\|^{2}+\|\hat{h}\|^{4}+4\left(K^{D}\right)^{2}\right) \sum_{r}\left(h_{2 k}^{r} \mathbf{x} \wedge e_{1} \wedge e_{r}-h_{1 k}^{r} \mathbf{x} \wedge e_{2} \wedge e_{r}\right)
\end{aligned}
$$




$$
\begin{aligned}
& +4 K^{D}\|\hat{h}\|_{k}^{2} \mathbf{x} \wedge e_{3} \wedge e_{4}+4 K^{D}\left(1+2\|\hat{h}\|^{2}\right) \\
& \times\left\{e_{k} \wedge e_{3} \wedge e_{4}-\sum_{j} h_{j k}^{3} \mathbf{x} \wedge e_{j} \wedge e_{4}+\sum_{j} h_{j k}^{4} \mathbf{x} \wedge e_{j} \wedge e_{3}\right\} \\
& -2 \sum_{j, r}\|\hat{h}\|_{j}^{2} h_{2 j}^{r}\left\{e_{k} \wedge e_{1} \wedge e_{r}+\omega_{1}^{2}\left(e_{k}\right) \mathbf{x} \wedge e_{2} \wedge e_{r}\right. \\
& \left.+\sum_{s} \omega_{r}^{s}\left(e_{k}\right) \mathbf{x} \wedge e_{1} \wedge e_{s}+\sum_{s} h_{1 k}^{s} \mathbf{x} \wedge e_{s} \wedge e_{r}\right\} \\
& +2 \sum_{j, r}\|\hat{h}\|_{j}^{2} h_{1 j}^{r}\left\{e_{k} \wedge e_{2} \wedge e_{r}-\omega_{1}^{2}\left(e_{k}\right) \mathbf{x} \wedge e_{1} \wedge e_{r}\right. \\
& \left.+\sum_{s} \omega_{r}^{s}\left(e_{k}\right) \mathbf{x} \wedge e_{2} \wedge e_{s}+\sum_{s} h_{2 k}^{s} \mathbf{x} \wedge e_{s} \wedge e_{r}\right\}
\end{aligned}
$$

Now, by comparing the coefficients of $e_{k} \wedge e_{3} \wedge e_{4}$ in (6.27), we obtain from (6.9) and (6.27) that $4\|\hat{h}\|^{2}=\lambda_{p}+\lambda_{q}-2$, which is constant. Hence, $M^{2}$ has constant Gauss curvature. Hence, $M^{2}$ is an open piece of Veronese surface according to a result of Kenmotsu [16]. This contradicts to Theorem 5.1.

Case (ii): $h_{11}^{4}=0$. We obtain from (6.14), (6.15), (6.19) and (6.20) that

$$
\begin{aligned}
& h_{11}^{3} \omega_{4}^{5}\left(e_{2}\right)+h_{12}^{4} \omega_{3}^{5}\left(e_{1}\right)=h_{11}^{3} \omega_{4}^{5}\left(e_{1}\right)-h_{12}^{4} \omega_{3}^{5}\left(e_{2}\right)=0, \\
& h_{11}^{3} \omega_{3}^{5}\left(e_{1}\right)+h_{12}^{4} \omega_{4}^{5}\left(e_{2}\right)=h_{11}^{3} \omega_{3}^{5}\left(e_{2}\right)-h_{12}^{4} \omega_{4}^{5}\left(e_{1}\right)=0, \\
& h_{11}^{3} \omega_{4}^{6}\left(e_{1}\right)-h_{12}^{4} \omega_{3}^{6}\left(e_{2}\right)=h_{11}^{3} \omega_{3}^{6}\left(e_{2}\right)-h_{12}^{4} \omega_{4}^{6}\left(e_{1}\right)=0 .
\end{aligned}
$$

Since $h_{11}^{3}, h_{12}^{4} \neq 0$, solving this system gives

$$
\omega_{3}^{5}\left(e_{1}\right)= \pm \omega_{4}^{5}\left(e_{2}\right), \omega_{3}^{5}\left(e_{2}\right)= \pm \omega_{4}^{5}\left(e_{1}\right), \omega_{3}^{6}\left(e_{2}\right)= \pm \omega_{4}^{6}\left(e_{1}\right) .
$$

If $\omega_{3}^{5}=\omega_{4}^{5}=\omega_{3}^{6}=\omega_{4}^{6}=0$, then by applying the same argument as Case (i), we know that $M^{2}$ is a minimal surface of constant Gauss curvature of a totally geodesic $S^{4} \subset S^{m-1}$, which leads to the same contradiction as Case (i). Hence, at least one of $\omega_{3}^{5}, \omega_{3}^{6}, \omega_{4}^{5}, \omega_{4}^{6}$ is nonzero. Therefore, (6.28) and (6.29) yield $h_{11}^{3}= \pm h_{12}^{4}$. So, after replacing $e_{4}$ by $-e_{4}$ if necessary, we find

$$
\begin{aligned}
& h_{11}^{3}=h_{12}^{4} \neq 0, h_{12}^{3}=h_{11}^{4}=h_{22}^{4}=\omega_{3}^{6}\left(e_{1}\right)=\omega_{4}^{6}\left(e_{2}\right)=0, \\
& \omega_{3}^{5}\left(e_{1}\right)=-\omega_{4}^{5}\left(e_{2}\right), \omega_{3}^{5}\left(e_{2}\right)=\omega_{4}^{5}\left(e_{1}\right), \omega_{3}^{6}\left(e_{2}\right)=\omega_{4}^{6}\left(e_{1}\right), \\
& \|\hat{h}\|^{2}=4\left(h_{11}^{3}\right)^{2}=-2 K^{D} .
\end{aligned}
$$

By considering $e_{1} \wedge e_{2} \wedge e_{r}$, we obtain from (6.7), (6.8), (6.9) and (6.30) that

$$
\begin{aligned}
0= & \sum_{j, r} K_{j}^{D}\left(h_{1 j}^{r} \delta_{1 k}+h_{2 j}^{r} \delta_{2 k}\right) e_{1} \wedge e_{2} \wedge e_{r} \\
& +\sum_{i} K_{k}^{D} h_{i k}^{4} e_{k} \wedge e_{i} \wedge e_{3}-\sum_{j} K_{j}^{D} h_{k k}^{3} e_{j} \wedge e_{k} \wedge e_{4} \\
& -\sum_{i, j} K_{j}^{D}\left\{h_{i j}^{3} e_{k} \wedge e_{i} \wedge e_{4}-h_{i j}^{4} e_{k} \wedge e_{i} \wedge e_{3}\right\}
\end{aligned}
$$


Combining this with (6.30) gives $K_{1}^{D}=K_{2}^{D}=0$. Thus, $K^{D}, h_{11}^{3}, h_{12}^{4}$ are constant. So, it follows from the last equation in (6.30) that the Gauss curvature of $M^{2}$ is also constant. Thus, we have $K \geq 0$ (cf. [15]). From these, we find

$$
h\left(e_{1}, e_{1}\right)=c e_{3}, h\left(e_{1}, e_{2}\right)=c e_{4}, h\left(e_{2}, e_{2}\right)=-c e_{3},
$$

for a nonzero constant $c$. By applying (6.32) and the equation of Codazzi, we find

$$
\omega_{3}^{4}=2 \omega_{1}^{2}
$$

By differentiating (6.33) and applying (6.30) and structure equations, we have

$$
\sum_{\alpha}\left\|\omega_{3}^{\alpha}\right\|^{2}=\sum_{\alpha}\left\|\omega_{4}^{\alpha}\right\|^{2}=6 c^{2}-2=\frac{3}{2}\|\hat{h}\|^{2}-2
$$

Hence, after using (6.34) and the constancy of $K$ (or equivalently $\|\hat{h}\|)$ and $K^{D}$, we know that $(6.7)$ and $(6.8)$ reduce to

$$
\begin{aligned}
e_{k} \tilde{\nu}= & \sum_{r}\left(h_{2 k}^{r} \mathbf{x} \wedge e_{1} \wedge e_{r}-h_{1 k}^{r} \mathbf{x} \wedge e_{2} \wedge e_{r}\right) \\
e_{k}(\Delta \tilde{\nu})= & \|\hat{h}\|^{2} \sum_{r}\left(h_{2 k}^{r} \mathbf{x} \wedge e_{1} \wedge e_{r}-h_{1 k}^{r} \mathbf{x} \wedge e_{2} \wedge e_{r}\right) \\
& -\|\hat{h}\|^{2}\left\{e_{k} \wedge e_{3} \wedge e_{4}-h_{k k}^{3} \mathbf{x} \wedge e_{k} \wedge e_{4}+\sum_{j} h_{j k}^{4} \mathbf{x} \wedge e_{j} \wedge e_{3}\right. \\
& \left.-\sum_{\alpha} \omega_{3}^{\alpha}\left(e_{k}\right) \mathbf{x} \wedge e_{4} \wedge e_{\alpha}+\sum_{\alpha} \omega_{4}^{\alpha}\left(e_{k}\right) \mathbf{x} \wedge e_{3} \wedge e_{\alpha}\right\} \\
e_{k}\left(\Delta^{2} \tilde{\nu}\right)= & -2\|\hat{h}\|^{2} \sum_{j, \alpha}\left(h_{i j}^{3} e_{k} \omega_{4}^{\alpha}\left(e_{j}\right)-h_{i j}^{4} e_{k} \omega_{3}^{\alpha}\left(e_{j}\right)\right) \mathbf{x} \wedge e_{i} \wedge e_{\alpha} \\
& -\|\hat{h}\|^{2} \sum_{j, \alpha} e_{k}\left(\left(\nabla_{e_{j}} \omega_{3}^{\alpha}\right) e_{j}-\omega_{3}^{4}\left(e_{j}\right) \omega_{4}^{\alpha}\left(e_{j}\right)\right. \\
& \left.-\sum_{\beta} \omega_{3}^{\beta}\left(e_{j}\right) \omega_{\alpha}^{\beta}\left(e_{j}\right)\right) \mathbf{x} \wedge e_{4} \wedge e_{\alpha} \\
& +\|\hat{h}\|^{2} \sum_{j, \alpha} e_{k}\left(\left(\nabla_{e_{j}} \omega_{4}^{\alpha}\right) e_{j}-\omega_{4}^{3}\left(e_{j}\right) \omega_{3}^{\alpha}\left(e_{j}\right)\right. \\
& \left.-\sum_{\beta} \omega_{4}^{\beta}\left(e_{j}\right) \omega_{\alpha}^{\beta}\left(e_{j}\right)\right) \mathbf{x} \wedge e_{3} \wedge e_{\alpha} \\
& -2\|\hat{h}\|^{2} \sum_{j, \alpha} e_{k}\left(\omega_{3}^{\alpha}\left(e_{j}\right)\right) e_{j} \wedge e_{4} \wedge e_{\alpha}
\end{aligned}
$$


430

BANG-YEN CHEN AND HUEI-SHYONG LUE

$+2\|\hat{h}\|^{2} \sum_{j, \alpha} e_{k}\left(\omega_{4}^{\alpha}\left(e_{j}\right)\right) e_{j} \wedge e_{3} \wedge e_{\alpha}$

+2||$\hat{h} \|^{4} \sum_{r}\left(h_{2 k}^{r} \mathbf{x} \wedge e_{1} \wedge e_{r}-h_{1 k}^{r} \mathbf{x} \wedge e_{2} \wedge e_{r}\right)$

-2||$\hat{h} \|^{2}\left(6 c^{2}-1+\|\hat{h}\|^{2}\right)\left\{e_{k} \wedge e_{3} \wedge e_{4}-h_{k k}^{3} \mathbf{x} \wedge e_{k} \wedge e_{4}\right.$

$\left.+\sum_{j} h_{j k}^{\mathbf{4}} \mathbf{x} \wedge e_{j} \wedge e_{3}-\sum_{\alpha} \omega_{3}^{\alpha}\left(e_{k}\right) \mathbf{x} \wedge e_{4} \wedge e_{\alpha}+\sum_{\alpha} \omega_{4}^{\alpha}\left(e_{k}\right) \mathbf{x} \wedge e_{3} \wedge e_{\alpha}\right\}$

$-\|\hat{h}\|^{2} \sum_{j, \alpha}\left(\left(\nabla_{e_{j}} \omega_{3}^{\alpha}\right) e_{j}-\omega_{3}^{4}\left(e_{j}\right) \omega_{4}^{\alpha}\left(e_{j}\right)-\sum_{\gamma} \omega_{3}^{\gamma}\left(e_{j}\right) \omega_{\alpha}^{\gamma}\left(e_{j}\right)\right)$

$\times\left\{e_{k} \wedge e_{4} \wedge e_{\alpha}-\sum_{i} h_{i k}^{4} \mathbf{x} \wedge e_{i} \wedge e_{\alpha}-\omega_{3}^{4}\left(e_{k}\right) \mathbf{x} \wedge e_{3} \wedge e_{\alpha}\right.$

$\left.+\sum_{\beta} \omega_{4}^{\beta}\left(e_{k}\right) \mathbf{x} \wedge e_{\beta} \wedge e_{\alpha}+\omega_{3}^{\alpha}\left(e_{k}\right) \mathbf{x} \wedge e_{3} \wedge e_{4}+\sum_{\beta} \omega_{\alpha}^{\beta}\left(e_{k}\right) \mathbf{x} \wedge e_{4} \wedge e_{\beta}\right\}$

$+\|\hat{h}\|^{2} \sum_{j, \alpha}\left(\left(\nabla_{e_{j}} \omega_{4}^{\alpha}\right) e_{j}-\omega_{4}^{3}\left(e_{j}\right) \omega_{3}^{\alpha}\left(e_{j}\right)-\sum_{\gamma} \omega_{4}^{\gamma}\left(e_{j}\right) \omega_{\alpha}^{\gamma}\left(e_{j}\right)\right)$

$\times\left\{e_{k} \wedge e_{3} \wedge e_{\alpha}-\sum_{i} h_{i k}^{3} \mathbf{x} \wedge e_{i} \wedge e_{\alpha}+\omega_{3}^{4}\left(e_{k}\right) \mathbf{x} \wedge e_{4} \wedge e_{\alpha}\right.$

$\left.+\sum_{\beta} \omega_{3}^{\beta}\left(e_{k}\right) \mathbf{x} \wedge e_{\beta} \wedge e_{\alpha}-\omega_{4}^{\alpha}\left(e_{k}\right) \mathbf{x} \wedge e_{3} \wedge e_{4}+\sum_{\beta} \omega_{\alpha}^{\beta}\left(e_{k}\right) \mathbf{x} \wedge e_{3} \wedge e_{\beta}\right\}$

$-2\|\hat{h}\|^{2} \sum_{j, \alpha}\left(h_{i j}^{3} \omega_{4}^{\alpha}\left(e_{j}\right)-h_{i j}^{4} \omega_{3}^{\alpha}\left(e_{j}\right)\right)\left\{e_{k} \wedge e_{i} \wedge e_{\alpha}+\sum_{\ell} \omega_{i}^{\ell}\left(e_{k}\right) \mathbf{x} \wedge e_{\ell} \wedge e_{\alpha}\right.$

$+\sum_{r} h_{i k}^{r} \mathbf{x} \wedge e_{r} \wedge e_{\alpha}-\omega_{3}^{\alpha}\left(e_{k}\right) \mathbf{x} \wedge e_{i} \wedge e_{3}-\omega_{4}^{\alpha}\left(e_{k}\right) \mathbf{x} \wedge e_{i} \wedge e_{4}$

$\left.+\sum_{\beta} \omega_{\alpha}^{\beta}\left(e_{k}\right) \mathbf{x} \wedge e_{i} \wedge e_{\beta}\right\}-2\|\hat{h}\|^{2} \sum_{j, \alpha} \omega_{3}^{\alpha}\left(e_{j}\right)\left\{\sum_{i} \omega_{j}^{i}\left(e_{k}\right) e_{i} \wedge e_{4} \wedge e_{\alpha}\right.$

$+h_{j k}^{3} e_{3} \wedge e_{4} \wedge e_{\alpha}+\omega_{3}^{\alpha}\left(e_{k}\right) e_{j} \wedge e_{3} \wedge e_{4}+\sum_{\beta} \omega_{\alpha}^{\beta}\left(e_{k}\right) e_{j} \wedge e_{4} \wedge e_{\beta}$

$\left.-\sum_{i} h_{i k}^{4} e_{j} \wedge e_{i} \wedge e_{\alpha}-\omega_{3}^{4}\left(e_{k}\right) e_{j} \wedge e_{3} \wedge e_{\alpha}-\sum_{\beta} \omega_{4}^{\beta}\left(e_{k}\right) e_{j} \wedge e_{\alpha} \wedge e_{\beta}\right\}$

$+2\|\hat{h}\|^{2} \sum_{j, \alpha} \omega_{4}^{\alpha}\left(e_{j}\right)\left\{\sum_{i} \omega_{j}^{i}\left(e_{k}\right) e_{i} \wedge e_{3} \wedge e_{\alpha}-h_{j k}^{4} e_{3} \wedge e_{4} \wedge e_{\alpha}\right.$

$-h_{k k}^{3} e_{j} \wedge e_{k} \wedge e_{\alpha}+\omega_{3}^{4}\left(e_{k}\right) e_{j} \wedge e_{4} \wedge e_{\alpha}-\sum_{\beta} \omega_{3}^{\beta}\left(e_{k}\right) e_{j} \wedge e_{\alpha} \wedge e_{\beta}$

$\left.-\omega_{4}^{\alpha}\left(e_{k}\right) e_{j} \wedge e_{3} \wedge e_{4}+\sum_{\beta} \omega_{\alpha}^{\beta}\left(e_{k}\right) e_{j} \wedge e_{3} \wedge e_{\beta}\right\}$ 
By considering $e_{1} \wedge e_{3} \wedge e_{4}$ and $e_{2} \wedge e_{3} \wedge e_{4},(6.9)$ and (6.35) give

$$
\begin{aligned}
& 2\left(6 c^{2}-1+\|\left.\hat{h}\right|^{2}\right) e_{k} \wedge e_{3} \wedge e_{4}+2 \sum_{j, \alpha} \omega_{3}^{\alpha}\left(e_{j}\right) \omega_{3}^{\alpha}\left(e_{k}\right) e_{j} \wedge e_{3} \wedge e_{4} \\
& +2 \sum_{j, \alpha} \omega_{4}^{\alpha}\left(e_{j}\right) \omega_{4}^{\alpha}\left(e_{k}\right) e_{j} \wedge e_{3} \wedge e_{4}=\left(\lambda_{p}+\lambda_{q}\right) e_{k} \wedge e_{3} \wedge e_{4} .
\end{aligned}
$$

Combining this with (6.30) and (6.32) gives

$$
\lambda_{p}+\lambda_{q}=8|| \hat{h} \|^{2}-6 .
$$

It follows from (6.9) and (6.35) that

$$
\begin{aligned}
0= & 2 \sum_{j, \alpha}\left(e_{k}\left(\omega_{4}^{\alpha}\left(e_{j}\right)\right)+\omega_{3}^{\alpha}\left(e_{j}\right) \omega_{3}^{4}\left(e_{k}\right)\right) e_{j} \wedge e_{3} \wedge e_{\alpha} \\
& +\sum_{j, \alpha}\left[\left(\nabla_{e_{j}} \omega_{4}^{\alpha}\right) e_{j}+\omega_{3}^{4}\left(e_{j}\right) \omega_{3}^{\alpha}\left(e_{j}\right)+\sum_{\gamma} \omega_{4}^{\gamma}\left(e_{j}\right) \omega_{\gamma}^{\alpha}\left(e_{j}\right)\right] e_{k} \wedge e_{3} \wedge e_{\alpha} \\
& +2 \sum_{i, j, \alpha} \omega_{4}^{\alpha}\left(e_{j}\right) \omega_{j}^{i}\left(e_{k}\right) e_{i} \wedge e_{3} \wedge e_{\alpha}+2 \sum_{j, \alpha, \beta} \omega_{4}^{\alpha}\left(e_{j}\right) \omega_{\alpha}^{\beta}\left(e_{k}\right) e_{j} \wedge e_{3} \wedge e_{\beta}
\end{aligned}
$$

and

(6.38)

$$
\begin{aligned}
0= & 2 \sum_{j, \alpha} e_{k}\left(\omega_{3}^{\alpha}\left(e_{j}\right)\right) e_{j} \wedge e_{4} \wedge e_{\alpha}-2 \sum_{j, \alpha} \omega_{4}^{\alpha}\left(e_{j}\right) \omega_{3}^{4}\left(e_{k}\right) e_{j} \wedge e_{4} \wedge e_{\alpha} \\
& +\sum_{j, \alpha}\left[\left(\nabla_{e_{j}} \omega_{3}^{\alpha}\right) e_{j}-\omega_{3}^{4}\left(e_{j}\right) \omega_{4}^{\alpha}\left(e_{j}\right)-\sum_{\gamma} \omega_{3}^{\gamma}\left(e_{j}\right) \omega_{\alpha}^{\gamma}\left(e_{j}\right)\right] e_{k} \wedge e_{4} \wedge e_{\alpha} \\
& +2 \sum_{i, j, \alpha} \omega_{3}^{\alpha}\left(e_{j}\right) \omega_{j}^{i}\left(e_{k}\right) e_{i} \wedge e_{4} \wedge e_{\alpha}+2 \sum_{j, \alpha, \beta} \omega_{3}^{\alpha}\left(e_{j}\right) \omega_{\alpha}^{\beta}\left(e_{k}\right) e_{j} \wedge e_{4} \wedge e_{\beta} .
\end{aligned}
$$

Now, by considering $e_{1} \wedge e_{3} \wedge e_{6}, e_{2} \wedge e_{3} \wedge e_{6}, e_{1} \wedge e_{4} \wedge e_{6}$, and $e_{2} \wedge e_{4} \wedge e_{6}$ respectively, we obtain from (6.37) and (6.38) that

$$
\begin{aligned}
e_{2}\left(\omega_{3}^{6}\left(e_{2}\right)\right) & =-\omega_{3}^{5}\left(e_{2}\right) \omega_{5}^{6}\left(e_{2}\right), \omega_{3}^{6}\left(e_{2}\right)\left(\omega_{1}^{2}\left(e_{1}\right)+\omega_{3}^{4}\left(e_{1}\right)\right) \\
& =\omega_{3}^{5}\left(e_{1}\right) \omega_{5}^{6}\left(e_{1}\right), \\
e_{1}\left(\omega_{3}^{6}\left(e_{2}\right)\right) & =-\omega_{3}^{5}\left(e_{2}\right) \omega_{5}^{6}\left(e_{1}\right), \omega_{3}^{6}\left(e_{2}\right)\left(\omega_{1}^{2}\left(e_{2}\right)+\omega_{3}^{4}\left(e_{2}\right)\right) \\
& =\omega_{3}^{5}\left(e_{1}\right) \omega_{5}^{6}\left(e_{2}\right) .
\end{aligned}
$$

Similarly, by considering $e_{i} \wedge e_{r} \wedge e_{5}$ for $i=1,2$ and $r=3,4$, we find

$$
\begin{aligned}
& e_{2}\left(\omega_{3}^{5}\left(e_{2}\right)\right)=\omega_{3}^{6}\left(e_{2}\right) \omega_{5}^{6}\left(e_{2}\right)-\omega_{3}^{5}\left(e_{1}\right)\left(\omega_{1}^{2}\left(e_{2}\right)+\omega_{3}^{4}\left(e_{2}\right)\right), \\
& e_{1}\left(\omega_{3}^{5}\left(e_{2}\right)\right)=\omega_{3}^{6}\left(e_{2}\right) \omega_{5}^{6}\left(e_{1}\right)-\omega_{3}^{5}\left(e_{1}\right)\left(\omega_{1}^{2}\left(e_{1}\right)+\omega_{3}^{4}\left(e_{1}\right)\right), \\
& e_{2}\left(\omega_{3}^{5}\left(e_{1}\right)\right)=\omega_{3}^{5}\left(e_{2}\right)\left(\omega_{1}^{2}\left(e_{2}\right)+\omega_{3}^{4}\left(e_{2}\right)\right), \\
& e_{1}\left(\omega_{3}^{5}\left(e_{1}\right)\right)=\omega_{3}^{5}\left(e_{2}\right)\left(\omega_{1}^{2}\left(e_{1}\right)+\omega_{3}^{4}\left(e_{1}\right)\right) .
\end{aligned}
$$


Also, by differentiating $\omega_{5}^{6}$ and applying (6.30), we find

$$
\begin{aligned}
e_{2}\left(\omega_{5}^{6}\left(e_{1}\right)\right)-e_{1}\left(\omega_{5}^{6}\left(e_{2}\right)\right)= & \omega_{1}^{2}\left(e_{1}\right) \omega_{5}^{6}\left(e_{1}\right)+\omega_{1}^{2}\left(e_{2}\right) \omega_{5}^{6}\left(e_{2}\right) \\
& +2 \omega_{3}^{5}\left(e_{1}\right) \omega_{3}^{6}\left(e_{2}\right)
\end{aligned}
$$

Now, by applying (6.30), (6.39)-(6.44), and a direct computation, we have

$$
\begin{aligned}
& \sum_{j}\left(h_{j k}^{3} \omega_{3}^{\alpha}\left(e_{j}\right)+h_{j k}^{4} \omega_{4}^{\alpha}\left(e_{j}\right)\right)=\sum_{j}\left(h_{i j}^{3} \omega_{4}^{\alpha}\left(e_{j}\right)-h_{i j}^{4} \omega_{3}^{\alpha}\left(e_{j}\right)\right)=0, \\
& \sum_{j}\left\{\left(\nabla_{e_{j}} \omega_{3}^{\alpha}\right) e_{j}-\omega_{3}^{4}\left(e_{j}\right) \omega_{4}^{\alpha}\left(e_{j}\right)-\sum_{\beta} \omega_{3}^{\beta}\left(e_{j}\right) \omega_{\alpha}^{\beta}\left(e_{j}\right)\right\}=0, \\
& \sum_{j}\left\{\left(\nabla_{e_{j}} \omega_{4}^{\alpha}\right) e_{j}-\omega_{4}^{3}\left(e_{j}\right) \omega_{3}^{\alpha}\left(e_{j}\right)-\sum_{\beta} \omega_{4}^{\beta}\left(e_{j}\right) \omega_{\alpha}^{\beta}\left(e_{j}\right)\right\}=0
\end{aligned}
$$

for $i, k=1,2 ; \alpha=5,6$. Thus, in views of $(6.24),(6.30),(6.37),(6.38)$ and $(6.46)$, equation $(6.35)$ reduces to

$$
\begin{aligned}
e_{k} \tilde{\nu}= & \sum_{r}\left(h_{2 k}^{r} \mathbf{x} \wedge e_{1} \wedge e_{r}-h_{1 k}^{r} \mathbf{x} \wedge e_{2} \wedge e_{r}\right) \\
e_{k}(\Delta \tilde{\nu})= & \|\hat{h}\|^{2} \sum_{r}\left(h_{2 k}^{r} \mathbf{x} \wedge e_{1} \wedge e_{r}-h_{1 k}^{r} \mathbf{x} \wedge e_{2} \wedge e_{r}\right) \\
& -\|\hat{h}\|^{2}\left\{\left(\delta_{1 k} e_{1} \wedge e_{3} \wedge e_{4}+\delta_{2 k} e_{2} \wedge e_{3} \wedge e_{4}\right)\right. \\
& +\sum_{j}\left(h_{j k}^{4}-h_{k k}^{3} \delta_{j k}\right) \mathbf{x} \wedge e_{j} \wedge e_{3} \\
& \left.-\sum_{\alpha} \omega_{3}^{\alpha}\left(e_{k}\right) \mathbf{x} \wedge e_{4} \wedge e_{\alpha}+\sum_{\alpha} \omega_{4}^{\alpha}\left(e_{k}\right) \mathbf{x} \wedge e_{3} \wedge e_{\alpha}\right\} \\
e_{k}\left(\Delta^{2} \tilde{\nu}\right)= & 2\|\hat{h}\|^{4} \sum_{r}\left(h_{2 k}^{r} \mathbf{x} \wedge e_{1} \wedge e_{r}-h_{1 k}^{r} \mathbf{x} \wedge e_{2} \wedge e_{r}\right) \\
& +2\|\hat{h}\|^{2}\left(2+\|\hat{h}\|^{2}\right)\left\{h_{k k}^{3} \mathbf{x} \wedge e_{k} \wedge e_{4}-\sum_{j} h_{j k}^{4} \mathbf{x} \wedge e_{j} \wedge e_{3}\right. \\
& \left.-e_{k} \wedge e_{3} \wedge e_{4}+\sum_{\alpha} \omega_{3}^{\alpha}\left(e_{k}\right) \mathbf{x} \wedge e_{4} \wedge e_{\alpha}-\sum_{\alpha} \omega_{4}^{\alpha}\left(e_{k}\right) \mathbf{x} \wedge e_{3} \wedge e_{\alpha}\right\} \\
& -2\|\hat{h}\|^{2}\left(\delta_{1 k} e_{1} \wedge e_{3} \wedge e_{4}+\delta_{2 k} e_{2} \wedge e_{3} \wedge e_{4}\right) .
\end{aligned}
$$

Now, by considering the coefficients of $\mathbf{x} \wedge e_{1} \wedge e_{3}$, we obtain from (4.2) and (6.47) that

$$
4\|\hat{h}\|^{4}-2\left(\lambda_{p}+\lambda_{q}-2\right)\|\hat{h}\|^{2}+\lambda_{p} \lambda_{q}=0 .
$$

Combining this with $(6.36)$ yields

$$
\lambda_{p}+\lambda_{q}=8\|\hat{h}\|^{2}-6, \quad \lambda_{p} \lambda_{q}=\left(9\|\hat{h}\|^{2}-10\right)\|\hat{h}\|^{2} .
$$

Case (i): $K>0$. In this case, a result of Wallach [22] implies that there exists an integer $k \geq 2$ such that $M^{2}$ is an open part of the $k$-th standard immersion 
$\psi_{k}: S\left(\frac{2}{k(k+1)}\right) \rightarrow S^{2 k} \subset S^{m-1}$ of the 2-sphere of constant curvature $\frac{2}{k(k+1)}$. Without loss generality, we may assume $M=S\left(\frac{2}{k(k+1)}\right)$.

It is known that eigenvalues of the Laplacian $\Delta$ on $S\left(\frac{2}{k(k+1)}\right)$ are given by

$$
\lambda_{i}=\frac{2 i(i+1)}{k(k+1)}, i=0,1,2, \ldots
$$

On the other hand, the equation of Gauss gives

$$
\|\hat{h}\|^{2}=\frac{2(k-1)(k+2)}{k(k+1)} .
$$

Substituting (6.50) into (6.48) yields

$$
\lambda_{p}, \lambda_{q}=\frac{5 k(k+1)-16 \mp \sqrt{112+k(1+k)\left(9 k^{2}+9 k-56\right)}}{k(k+1)} .
$$

By comparing (6.49) and (6.51) we find

$$
\begin{aligned}
& 5 k(k+1)-\sqrt{112+k(1+k)\left(9 k^{2}+9 k-56\right)}=2 i(i+1)+16, \\
& 5 k(k+1)+\sqrt{112+k(1+k)\left(9 k^{2}+9 k-56\right)}=2 j(j+1)+16
\end{aligned}
$$

for some natural numbers $i, j$, which is impossible unless $k=2, i=1$ and $j=3$. It follows from $k=2$ that the minimal surface is the Veronese surface. So, according to Theorem 4.1 the spherical Gauss map is mass-symmetric. This is a contradiction.

Case (ii): $K=0$. In this case, equation of Gauss yields $\|\hat{h}\|^{2}=2$. Hence, we obtain from (6.48) that $\lambda_{p}=2$ and $\lambda_{q}=8$. Combining these with (6.30) yields

$$
\begin{aligned}
& h_{11}^{3}=h_{12}^{4}=\frac{1}{\sqrt{2}}, h_{12}^{3}=h_{11}^{4}=h_{22}^{4}=\omega_{3}^{6}\left(e_{1}\right)=\omega_{4}^{6}\left(e_{2}\right)=0, \\
& \omega_{3}^{5}\left(e_{1}\right)=-\omega_{4}^{5}\left(e_{2}\right), \omega_{3}^{5}\left(e_{2}\right)=\omega_{4}^{5}\left(e_{1}\right), \omega_{3}^{6}\left(e_{2}\right)=\omega_{4}^{6}\left(e_{1}\right), \\
& K^{D}=-1,\|\hat{h}\|^{2}=2 .
\end{aligned}
$$

Since $K=0$, there exists an integer $\ell \geq 2$ such that $M^{2}$ is an open portion of a flat minimal torus $T^{2}$ of a totally geodesic $S^{2 \ell-1} \subset S^{m}$ (see, [14]). Also, we see from Theorem 4.1 that $\ell \geq 3$. We many assume that the $T^{2}$ is $\mathbb{E}^{2} / \Lambda$, where $\Lambda$ is the lattice in $\mathbb{E}^{2}$ defined by

$$
\Lambda=\left\{\left(2 n_{1} \pi u, 2 n_{2} \pi v+2 h \pi w\right): n_{1}, n_{2} \in \mathbb{Z}\right\}
$$

for real numbers $u, v, w$ with $u, v>0$. It is known that the dual lattice of $\Lambda$ is

$$
\Lambda^{*}=\left\{\left(\frac{m_{1}}{2 \pi u}-\frac{m_{2} w}{2 \pi u v}, \frac{m_{2}}{2 \pi v}\right): m_{1}, m_{2} \in \mathbb{Z}\right\},
$$

the spectrum of $T^{2}=\mathbb{E}^{2} / \Lambda$ is

$$
\left\{\left(\frac{m_{1}}{u}-\frac{m_{2} w}{u v}\right)^{2}+\left(\frac{m_{2}}{v}\right)^{2}: m_{1}, m_{2} \in \mathbb{Z}\right\}
$$


and the eigenspace $V(\lambda)$ of $\Delta$ with eigenvalue $\lambda$ is

$$
\begin{gathered}
\operatorname{Span}\left\{\cos \left(\frac{\alpha s}{u}+\frac{n t}{v}\right), \sin \left(\frac{\alpha s}{u}+\frac{n t}{v}\right):\left(\frac{\alpha}{u}\right)^{2}+\left(\frac{n}{v}\right)^{2}=\lambda\right\}, \\
\alpha=m_{1}-m_{2} \frac{w}{v} .
\end{gathered}
$$

Put $\mathcal{L}=\{1,2, \ldots, \ell\}$ with $\ell \geq 3$. Since $T^{2}$ is a minimal surface of $S^{2 \ell-1}$, we have $\Delta \mathbf{x}=2 \mathbf{x}$. We may assume that $\mathbf{x}: T^{2} \rightarrow S^{2 \ell-1} \subset \mathbb{E}^{2 \ell}$ is given by

$$
\mathbf{x}(s, t)=\left(\mu_{i} \sin \left(\bar{\alpha}_{i} s+\bar{p}_{i} t\right), \mu_{i} \cos \left(\bar{\alpha}_{i} s+\bar{p}_{i} t\right)\right)_{i \in \mathcal{L}}
$$

with $\mu_{i}>0, \bar{p}_{i} \geq 0$ and $\bar{\alpha}_{i} \in \mathbf{R}$ satisfying $\bar{\alpha}_{i}^{2}+\bar{p}_{i}^{2}=2$. From (6.58) we get

$$
\begin{aligned}
& \frac{\partial \mathbf{x}}{\partial s}=\left(\bar{\alpha}_{i} \mu_{i} \cos \left(\bar{\alpha}_{i} s+\bar{p}_{i} t\right),-\bar{\alpha}_{i} \mu_{i} \sin \left(\bar{\alpha}_{i} s+\bar{p}_{i} t\right)\right)_{i \in \mathcal{L}}, \\
& \frac{\partial \mathbf{x}}{\partial t}=\left(\bar{p}_{i} s \mu_{i} \cos \left(\bar{\alpha}_{i} s+\bar{p}_{i} t\right),-\bar{p}_{i} \mu_{i} \sin \left(\bar{\alpha}_{i} s+\bar{p}_{i} t\right)\right)_{i \in \mathcal{L}}
\end{aligned}
$$

$\partial \mathbf{x} / \partial s$ and $\partial \mathbf{x} / \partial t$ are orthonormal vector fields on $T^{2}$.

Since $\mathbf{x}$ is isometric, (6.59) gives

$$
\sum_{i} \mu_{i}^{2}=\sum_{i} \mu^{2} \bar{\alpha}_{i}^{2}=\sum_{i} \mu_{i}^{2} \bar{p}_{i}^{2}=1, \quad \sum_{i} \mu_{i}^{2} \bar{\alpha}_{i}^{2} \bar{p}_{i}^{2}=0
$$

It follows from the definition of $\tilde{\nu},(6.58),(6.59)$, and properties of $\wedge$ that each coordinate function of $\tilde{\nu}$ in $\mathbb{E}^{\left(\begin{array}{c}m \\ 3\end{array}\right)}$ is a multiple of one of the following functions:

$$
\begin{aligned}
& \cos \left(\bar{\alpha}_{i} s+\bar{p}_{i} t\right), \sin \left(\bar{\alpha}_{i} s+\bar{p}_{i} t\right) \sin \left(\bar{\alpha}_{j} s+\bar{p}_{j} t\right) \sin \left(\bar{\alpha}_{k} s+\bar{p}_{k} t\right), \\
& \sin \left(\bar{\alpha}_{i} s+\bar{p}_{i} t\right), \sin \left(\bar{\alpha}_{i} s+\bar{p}_{i} t\right) \sin \left(\bar{\alpha}_{j} s+\bar{p}_{j} t\right) \cos \left(\bar{\alpha}_{k} s+\bar{p}_{k} t\right), \\
& \sin \left(\bar{\alpha}_{i} s+\bar{p}_{i} t\right) \cos \left(\bar{\alpha}_{j} s+\bar{p}_{j} t\right) \cos \left(\bar{\alpha}_{k} s+\bar{p}_{k} t\right), \\
& \cos \left(\bar{\alpha}_{i} s+\bar{p}_{i} t\right) \cos \left(\bar{\alpha}_{j} s+\bar{p}_{j} t\right) \cos \left(\bar{\alpha}_{k} s+\bar{p}_{k} t\right)
\end{aligned}
$$

for distinct $i, j, k \in \mathcal{L}$. Thus, each coordinate function of $\tilde{p}$ in $\mathbb{E}^{\left(\begin{array}{c}m \\ 3\end{array}\right)}$ is a linear combination of

$$
\begin{aligned}
& \cos \left(\left(\bar{\alpha}_{i}+\bar{\alpha}_{j}+\bar{\alpha}_{k}\right) s+\left(\bar{p}_{i}+\bar{p}_{j}+\bar{p}_{k}\right) t\right), \\
& \sin \left(\left(\bar{\alpha}_{i}+\bar{\alpha}_{j}+\bar{\alpha}_{k}\right) s+\left(\bar{p}_{i}+\bar{p}_{j}+\bar{p}_{k}\right) t\right), \\
& \cos \left(\left(\bar{\alpha}_{i}+\bar{\alpha}_{j}-\bar{\alpha}_{k}\right) s+\left(\bar{p}_{i}+\bar{p}_{j}-\bar{p}_{k}\right) t\right), \\
& \sin \left(\left(\bar{\alpha}_{i}+\bar{\alpha}_{j}-\bar{\alpha}_{k}\right) s+\left(\bar{p}_{i}+\bar{p}_{j}-\bar{p}_{k}\right) t\right), \\
& \cos \left(\bar{\alpha}_{i} s+\bar{p}_{i} t\right), \sin \left(\bar{\alpha}_{i} s+\bar{p}_{i} t\right)
\end{aligned}
$$

for distinct $i, j, k \in \mathcal{L}$.

Since we have a spectral resolution: $\tilde{\nu}=c+\tilde{\nu}_{1}+\tilde{\nu}_{2}$ with $\Delta \tilde{\nu}_{1}=2 \tilde{\nu}_{1}$, $\Delta \tilde{\nu}_{2}=8 \tilde{\nu}_{2}$, and $0 \neq c \in \mathbb{E}^{\left(\begin{array}{c}m \\ 3\end{array}\right)}$, the above observation gives the conditions:

$$
\begin{aligned}
& \left(\bar{\alpha}_{i}+\bar{\alpha}_{j}+\bar{\alpha}_{k}\right)^{2}+\left(\bar{p}_{i}+\bar{p}_{j}+\bar{p}_{k}\right)^{2},\left(\bar{\alpha}_{i}+\bar{\alpha}_{j}-\bar{\alpha}_{k}\right)^{2}+\left(\bar{p}_{i}+\bar{p}_{j}-\bar{p}_{k}\right)^{2} \\
\in & \{0,2,8\}
\end{aligned}
$$


for distinct $i, j, k \in \mathcal{L}$. By combining this with $\bar{\alpha}_{i}^{2}+\bar{p}_{i}^{2}=2$, we find

$$
\gamma_{i j}+\gamma_{i k}+\gamma_{j k}, \gamma_{i j}-\gamma_{i k}-\gamma_{j k} \in\{-3,-2,1\}
$$

for distinct $i, j, k \in \mathcal{L}$, where $\gamma_{i j}=\bar{\alpha}_{i} \bar{\alpha}_{j}+\bar{p}_{i} \bar{p}_{j}$. Condition (6.63) yields

$$
\gamma_{i j} \in\left\{-3,-\frac{5}{2},-2,-1,-\frac{1}{2}, 1\right\}
$$

for distinct $i, j \in \mathcal{L}$. From condition (6.64) we find

$$
\gamma_{i j}+\gamma_{k \ell} \in\left\{-6,-\frac{11}{2},-5,-\frac{9}{2},-4,-\frac{7}{2},-3,-\frac{5}{2}-2,-\frac{3}{2},-1,0, \frac{1}{2}, 2\right\} .
$$

We divide the proof of Case (ii) into two cases.

Case (ii-a): Two of $\gamma_{i j}$ are equal. Without loss of generality, we may assume that either (1) $\gamma_{12}=\gamma_{13}$ or $(2) \gamma_{12}=\gamma_{34} \neq \gamma_{13}$.

Case (ii-a-1): $\gamma_{12}=\gamma_{13}$. In this case, (6.64) and condition $\gamma_{12}-\gamma_{13}-\gamma_{23} \in$ $\{-3,-2,1\}$ from $(6.63)$ give

$$
\gamma_{23}=-1 \text { and } \gamma_{12}=\gamma_{13} \in\left\{-3,-\frac{5}{2},-2,-1,-\frac{1}{2}, 1\right\} .
$$

If $\gamma_{12}=\gamma_{13} \in\left\{-3,-\frac{5}{2},-2\right\}$, we obtain from $(6.66)$ that $\gamma_{12}+\gamma_{13}+\gamma_{23} \leq-5$ which contradicts to (6.63). So, this is impossible.

If $\gamma_{12}=\gamma_{13}=-\frac{1}{2}$ holds, then (6.66) yields $\gamma_{23}-\gamma_{12}-\gamma_{13}=0$, which contradicts to (6.63). Thus, this is also impossible. Consequently, we must have either (1) $\gamma_{12}=\gamma_{13}=-1$ or (2) $\gamma_{12}=\gamma_{13}=1$.

First, let us assume that $\ell \geq 4$.

If $\gamma_{12}=\gamma_{13}=-1$ holds, then condition $\gamma_{12}+\gamma_{14}+\gamma_{24} \in\{-3,-2,1\}$ gives $\gamma_{14}+\gamma_{24} \in\{-2,-1,2\}$. Also, we obtain from $\gamma_{12}-\gamma_{14}-\gamma_{24} \in\{-3,-2,1\}$ that $\gamma_{14}+\gamma_{24} \in\{-2,1,2\}$. By combining these we obtain $\gamma_{14}+\gamma_{24} \in\{-2,2\}$.

Similarly, using $\gamma_{12}=\gamma_{23}=-1$ and $\gamma_{13}=\gamma_{23}=-1$ we obtain $\gamma_{14}+\gamma_{34} \in$ $\{-2,2\}$ and $\gamma_{24}+\gamma_{34} \in\{-2,2\}$, respectively. Thus, we know that $\gamma_{14}+$ $\gamma_{24}, \gamma_{14}+\gamma_{34}$ and $\gamma_{24}+\gamma_{34}$ belong to $\{-2,2\}$. After solving this system under the two side conditions: (6.63) and (6.64), we obtain either $\gamma_{14}=\gamma_{24}=\gamma_{34}=1$ or $\gamma_{14}=\gamma_{24}=\gamma_{34}=-1$.

If $\gamma_{12}=\gamma_{13}=-1$ holds, we have either

(a) $\gamma_{i j}=-1,1 \leq i<j \leq 4$; or

(b) $\gamma_{12}=\gamma_{13}=\gamma_{23}=-1$ and $\gamma_{14}=\gamma_{24}=\gamma_{34}=1$.

Next, assume that $\gamma_{12}=\gamma_{13}=1$ holds. If $\gamma_{14} \in\left\{-3,-\frac{5}{2},-2\right\}$, then condition $\gamma_{12}-\gamma_{14}-\gamma_{24} \in\{-3,-2,1\}$ from (6.63) implies $\gamma_{24} \geq 2$. This contradicts to $(6.64)$.

If $\gamma_{14}=-\frac{1}{2}$, then condition $\gamma_{12}-\gamma_{14}-\gamma_{24} \in\{-3,-2,1\}$ from (6.63) and (6.64) imply $\gamma_{24} \in\left\{\frac{1}{2}, \frac{7}{2}, \frac{9}{2}\right\}$, which contradicts to (6.64).

If $\gamma_{14}=1$, condition $\gamma_{12}+\gamma_{14}+\gamma_{24} \in\{-3,-2,1\}$ and (6.64) give $\gamma_{24}=-1$. From $\gamma_{23}-\gamma_{12}-\gamma_{13} \in\{-3,-2,1\}$ and $(6.64)$ we get $\gamma_{23}=-1$. Similarly, from $\gamma_{34}-\gamma_{24}-\gamma_{23} \in\{-3,-2,1\}$ and $(6.64)$, we get $\gamma_{34}=-1$. Therefore, we have $\gamma_{12}=\gamma_{13}=\gamma_{14}=1$ and $\gamma_{23}=\gamma_{24}=\gamma_{34}=-1$.

If $\gamma_{14}=-1$, then $\gamma_{12}-\gamma_{14}-\gamma_{24} \in\{-3,-2,1\}$ and (6.64) yield $\gamma_{24}=1$. Also, (6.64) and $\gamma_{13}-\gamma_{14}-\gamma_{34} \in\{-3,-2,1\}$ from (6.63) give $\gamma_{34}=1$. Hence, 
we obtain $\gamma_{12}=\gamma_{13}=\gamma_{24}=\gamma_{34}=1, \quad \gamma_{23}=\gamma_{14}=-1$. Therefore, under $\gamma_{12}=\gamma_{13}$, one of the following two cases must occurs:

(A): $\ell \geq 4$ and $\left(\gamma_{12}, \gamma_{13}, \gamma_{14}, \gamma_{23}, \gamma_{24}, \gamma_{34}\right)$ is one of the following:

$$
\begin{aligned}
& (-1,-1,-1,-1,-1,-1), \quad(-1,-1,1,-1,1,1), \\
& (1,1,-1,-1,1,1), \quad(1,1,1,-1,-1,-1)
\end{aligned}
$$

(B): $\ell=3$ and $\gamma_{12}=\gamma_{13}=1$ or $\gamma_{12}=\gamma_{13}=-1$.

Now, we claim that (A) cannot happen. This can be seen as follows:

If $\left(\gamma_{12}, \gamma_{13}, \gamma_{14}, \gamma_{23}, \gamma_{24}, \gamma_{34}\right)$ is given by the first or second case, then we get $\gamma_{12}=\gamma_{13}=\gamma_{23}=-1$. In this case, we find $\bar{\alpha}_{1} \bar{\alpha}_{2}+\bar{p}_{1} \bar{p}_{2}=\bar{\alpha}_{1} \bar{\alpha}_{3}+\bar{p}_{1} \bar{p}_{3}=$ $\bar{\alpha}_{2} \bar{\alpha}_{3}+\bar{p}_{2} \bar{p}_{3}=-1$. After solving this for $\bar{\alpha}_{1}$, we get

$$
\bar{\alpha}_{1}=\frac{\sqrt{\left(1+\bar{p}_{1} \bar{p}_{2}\right)\left(1+\bar{p}_{1} \bar{p}_{3}\right)}}{\sqrt{-\left(1+\bar{p}_{2} \bar{p}_{3}\right)}} \text { or }=\frac{\sqrt{-\left(1+\bar{p}_{1} \bar{p}_{2}\right)\left(1+\bar{p}_{1} \bar{p}_{3}\right)}}{\sqrt{1+\bar{p}_{2} \bar{p}_{3}}} \text {. }
$$

But this is impossible, since $\bar{p}_{1}, \bar{p}_{2}, \bar{p}_{3}$ are $\geq 0$ and $\bar{\alpha}_{1}$ is a real number.

Now, assume $\left(\gamma_{12}, \gamma_{13}, \gamma_{14}, \gamma_{23}, \gamma_{24}, \gamma_{34}\right)=(1,1,-1,-1,1,1)$. We have

$$
\begin{aligned}
& \bar{\alpha}_{1} \bar{\alpha}_{2}+\bar{p}_{1} \bar{p}_{2}=\bar{\alpha}_{1} \bar{\alpha}_{3}+\bar{p}_{1} \bar{p}_{3}=1, \\
& \bar{\alpha}_{2} \bar{\alpha}_{3}+\bar{p}_{2} \bar{p}_{3}=\bar{\alpha}_{1} \bar{\alpha}_{4}+\bar{p}_{1} \bar{p}_{4}=-1, \\
& \bar{\alpha}_{2} \bar{\alpha}_{4}+\bar{p}_{2} \bar{p}_{4}=\bar{\alpha}_{3} \bar{\alpha}_{4}+\bar{p}_{3} \bar{p}_{4}=1 .
\end{aligned}
$$

After solving system (6.69), we obtain

$$
\begin{aligned}
&\left(\bar{\alpha}_{1}, \bar{\alpha}_{2}, \bar{\alpha}_{3}, \bar{\alpha}_{4}\right)= \pm\left(\frac{\sqrt{\left(\bar{p}_{1} \bar{p}_{2}-1\right)\left(1-\bar{p}_{1} \bar{p}_{3}\right)}}{\sqrt{1+\bar{p}_{2} \bar{p}_{3}}},\right. \\
& \frac{-\sqrt{\left(\bar{p}_{1} \bar{p}_{2}-1\right)\left(1+\bar{p}_{2} \bar{p}_{3}\right)}}{\sqrt{1-\bar{p}_{2} \overline{\bar{p}}_{3}}}, \frac{\sqrt{\left(1-\bar{p}_{1} \bar{p}_{3}\right)\left(1+\bar{p}_{2} \bar{p}_{3}\right)}}{\sqrt{\bar{p}_{1} \bar{p}_{2}-1}}, \\
&\left.\frac{-\sqrt{1+\bar{p}_{2} \bar{p}_{3}}\left(1+\bar{p}_{1} \bar{p}_{4}\right)}{\sqrt{\left(1-\bar{p}_{1} \bar{p}_{2}\right)\left(\bar{p}_{1} \bar{p}_{3}-1\right)}}\right)
\end{aligned}
$$

Substituting this into $(6.70)$ gives $\left(\bar{p}_{1}+\bar{p}_{2}\right)\left(\bar{p}_{3}+\bar{p}_{4}\right)=\left(\bar{p}_{1}+\bar{p}_{3}\right)\left(\bar{p}_{2}+\bar{p}_{4}\right)=0$. Thus, one of the following four cases occurs:
$(\alpha) \bar{p}_{3}=\bar{p}_{2}=-\bar{p}_{1}$
( $\beta) \bar{p}_{4}=\bar{p}_{1}=-\bar{p}_{3}$
( $\gamma) \bar{p}_{3}=\bar{p}_{2}=-\bar{p}_{4}$
( $\delta) \bar{p}_{4}=\bar{p}_{1}=-\bar{p}_{2}$;

If $(\alpha)$ occurs (respectively, $(\beta),(\gamma)$, or $(\delta)$ occurs), we obtain

$$
\begin{aligned}
\bar{\alpha}_{1}= & \pm \sqrt{-\left(1+\bar{p}_{1}^{2}\right)}\left(\text { respectively, } \pm \sqrt{-\left(1+\bar{p}_{3}^{2}\right)},\right. \\
& \left. \pm \frac{\sqrt{-\left(1+\bar{p}_{1}^{2}\right)\left(1+\bar{p}_{1} \bar{p}_{4}\right)}}{\sqrt{1+\bar{p}_{4}^{2}}} \text { or } \pm \frac{\sqrt{-\left(1+\bar{p}_{2} \bar{p}_{3}\right)\left(1+\bar{p}_{2}^{2}\right)}}{\sqrt{1+\bar{p}_{2} \bar{p}_{3}}}\right),
\end{aligned}
$$

which is a contradiction since $\bar{\alpha}_{1}$ is a real number. 
If $\left(\gamma_{12}, \gamma_{13}, \gamma_{14}, \gamma_{23}, \gamma_{24}, \gamma_{34}\right)=(1,1,1,-1,-1,-1)$ holds, then we get

$$
\begin{aligned}
& \bar{\alpha}_{1} \bar{\alpha}_{2}+\bar{p}_{1} \bar{p}_{2}=\bar{\alpha}_{1} \bar{\alpha}_{3}+\bar{p}_{1} \bar{p}_{3}=\bar{\alpha}_{1} \bar{\alpha}_{4}+\bar{p}_{1} \bar{p}_{4}=1, \\
& \bar{\alpha}_{2} \bar{\alpha}_{3}+\bar{p}_{2} \bar{p}_{3}=\bar{\alpha}_{2} \bar{\alpha}_{4}+\bar{p}_{2} \bar{p}_{4}=\bar{\alpha}_{3} \bar{\alpha}_{4}+\bar{p}_{3} \bar{p}_{4}=-1 .
\end{aligned}
$$

After solving this system, we get

$$
\bar{\alpha}_{2}=\frac{\sqrt{\left(1+\bar{p}_{2} \bar{p}_{3}\right)\left(1+\bar{p}_{2} \bar{p}_{4}\right)}}{\sqrt{-\left(1+\bar{p}_{3} \bar{p}_{4}\right)}} .
$$

But this is impossible since $\bar{\alpha}_{2}$ is a real number. So, (A) cannot happen. Consequently, under the assumption: $\gamma_{12}=\gamma_{13}$, we must have $\ell=3$ and $\gamma_{12}=\gamma_{13}= \pm 1$.

Case (ii-a-2): $\gamma_{12}=\gamma_{34} \neq \gamma_{13}$. This case occurs only when $\ell \geq 4$.

In view of (6.64), we divide this into six cases:

Case (ii-a-2.1): $\gamma_{12}=\gamma_{34}=-3$. In this case, $\gamma_{12}+\gamma_{13}+\gamma_{23} \in\{-3,-2,1\}$ implies $\gamma_{13}+\gamma_{23} \in\{0,1,4\}$. Combining this with (6.65) gives $\gamma_{13}+\gamma_{23}=0$. In view of $(6.64)$, we obtain $\gamma_{23}=1$ and $\gamma_{13}=-1$ from $\gamma_{13}-\gamma_{23}-\gamma_{12} \in$ $\{-3,-2,1\}$. Thus, we find $\gamma_{23}-\gamma_{13}-\gamma_{12}=5$ which contradicts to (6.63).

Case (ii-a-2.2): $\gamma_{12}=\gamma_{34}=-\frac{5}{2}$. Condition $\gamma_{12}+\gamma_{13}+\gamma_{23} \in\{-3,-2,1\}$ gives $\gamma_{13}+\gamma_{23} \in\left\{-\frac{1}{2}, \frac{1}{2}, \frac{7}{2}\right\}$. Combining this with (6.65) yields $\gamma_{13}+\gamma_{23}=\frac{1}{2}$. Thus, we obtain $\gamma_{23}=1$ and $\gamma_{13}=-\frac{1}{2}$ from $\gamma_{13}-\gamma_{23}-\gamma_{12} \in\{-3,-2,1\}$ using (6.64). From these we find $\gamma_{23}-\gamma_{13}-\gamma_{12}=4$ which is impossible.

Case (ii-a-2.3): $\gamma_{12}=\gamma_{34}=-2$. Condition $\gamma_{12}+\gamma_{13}+\gamma_{23} \in\{-3,-2,1\}$ gives $\gamma_{13}+\gamma_{23} \in\{-1,0,3\}$. Combining this with (6.65) yields $\gamma_{13}+\gamma_{23}=-1$ or 0 .

If $\gamma_{13}+\gamma_{23}=-1$ occurs, $\gamma_{13}-\gamma_{23}-\gamma_{12} \in\{-3,-2,1\}$ gives $\gamma_{23} \in\left\{0, \frac{3}{2}, 2\right\}$ which is impossible due to (6.64).

If $\gamma_{13}+\gamma_{23}=0$ occurs, $\gamma_{13}-\gamma_{23}-\gamma_{12} \in\{-3,-2,1\}$ gives $\gamma_{23} \in\left\{\frac{1}{2}, 2, \frac{5}{2}\right\}$, which is also impossible due to (6.64).

Case (ii-a-2.4): $\gamma_{12}=\gamma_{34}=-1$. In this case, $\gamma_{12}+\gamma_{13}+\gamma_{23} \in\{-3,-2,1\}$ yields $\gamma_{13}+\gamma_{23} \in\{-2,-1,2\}$.

If $\gamma_{13}+\gamma_{23}=-2$, condition $\gamma_{13}-\gamma_{23}-\gamma_{12} \in\{-3,-2,1\}$ implies that $\left(\gamma_{23}, \gamma_{13}\right)=(-1,-1),\left(-\frac{1}{2},-\frac{3}{2}\right)$, or $(1,-3)$. From these we have $\gamma_{23}-\gamma_{13}-$ $\gamma_{12} \in\{1,2,5\}$. Combining this with (6.63) yields $\gamma_{12}=\gamma_{13}=-1$, which contradicts to the assumption of Case (ii-a-1.2).

If $\gamma_{13}+\gamma_{23}=-1$ holds, $\gamma_{13}-\gamma_{23}-\gamma_{12} \in\left\{-\frac{5}{2},-\frac{3}{2}, \frac{3}{2}\right\}$ yields $\gamma_{23} \in\left\{-\frac{1}{2}, 1, \frac{3}{2}\right\}$. Comparing this with $(6.64)$ gives $\left(\gamma_{23}, \gamma_{13}\right)=\left(-\frac{1}{2},-\frac{1}{2}\right)$ or $(1,-2)$. The first case reduces to Case (ii-a-1) after making the interchanging: $1 \leftrightarrow 3$. If the later case holds, we find $\gamma_{23}-\gamma_{13}-\gamma_{12}=4$ which contradicts to (6.63).

If $\gamma_{13}+\gamma_{23}=2$ holds, $\gamma_{13}-\gamma_{23}-\gamma_{12} \in\{-3,-2,1\}$ implies that $\gamma_{23}=1, \frac{5}{2}$, or 3. Comparing this with (6.64) gives $\gamma_{23}=\gamma_{13}=1$. Thus, this case also reduces to Case (ii-a-1) after making the interchanging: $1 \leftrightarrow 3$.

Case (ii-a-2.5): $\gamma_{12}=\gamma_{34}=-\frac{1}{2}$. Condition $\gamma_{12}+\gamma_{13}+\gamma_{23} \in\{-3,-2,1\}$ gives $\gamma_{13}+\gamma_{23} \in\left\{-\frac{5}{2},-\frac{3}{2}, \frac{3}{2}\right\}$. Combining this with (6.65) yields $\gamma_{13}+\gamma_{23}=-\frac{5}{2}$ 
or $-\frac{3}{2}$. If $\gamma_{13}+\gamma_{23}=-\frac{5}{2}$ holds, we obtain $\left(\gamma_{23}, \gamma_{13}\right)=\left(-\frac{3}{2},-1\right)\left(0,-\frac{5}{2}\right)$, or $\left(\frac{1}{2},-3\right)$ from $\gamma_{13}-\gamma_{23}-\gamma_{12} \in\{-3,-2,1\}$. From these we know that $\gamma_{23}-\gamma_{13}-\gamma_{12}=0,3$, or 4 , which contradicts to (6.63).

If $\gamma_{13}+\gamma_{23}=-\frac{3}{2}$ holds, we get from $\gamma_{13}-\gamma_{23}-\gamma_{12} \in\{-3,-2,1\}$ that $\left(\gamma_{23}, \gamma_{13}\right)=\left(1,-\frac{5}{2}\right)\left(\frac{1}{2},-2\right)$, or $\left(-1,-\frac{1}{2}\right)$. Thus, we get $\gamma_{23}-\gamma_{13}-\gamma_{12}=4,3$, or 0 , respectively. This contradicts to (6.63), too.

Case (ii-a-2.6): $\gamma_{12}=\gamma_{34}=1$. In this case, $\gamma_{12}+\gamma_{13}+\gamma_{23} \in\{-3,-2,1\}$ gives $\gamma_{13}+\gamma_{23}=-4,-3$, or 0 . Thus we get $\gamma_{12}-\gamma_{13}-\gamma_{23}=5,4$ or 1 . Comparing this with (6.63) shows that $\gamma_{13}+\gamma_{23}=0$.

If $\gamma_{13}>0$ holds, we get $\gamma_{13}=1$ according to (6.64). This contradicts to the assumption: $\gamma_{12} \neq \gamma_{13}$ of Case (ii-a-1.2). Hence, we have $\gamma_{13}<0$ instead. Thus, according to $\gamma_{13}=-\gamma_{23}$ and (6.64), we have $\gamma_{23}=1$. So, we get $\gamma_{23}=-\gamma_{13}=1$. Consequently, we have $\gamma_{12}=\gamma_{23}=1$. Hence, this case reduces to Case (ii-a-1) after making the interchanging: $1 \leftrightarrow 2$.

Case (ii-b): $\gamma_{i j}$ are distinct for $1 \leq i<j \leq \ell$. Since $\gamma_{12}, \gamma_{13}, \gamma_{14}, \gamma_{23}, \gamma_{24}$, and $\gamma_{34}$ are distinct, (6.64) implies $\ell \leq 4$.

If $\ell=4$, then without loss of generality, we may put $\gamma_{12}=-3$. Then there exist $i, j, k, \ell \in\{1,2,3,4\}$ such that $\left(\gamma_{i j}, \gamma_{k \ell}\right)=(-2,-1)$ according to (6.64). So, we have $\gamma_{12}+\gamma_{i j}+\gamma_{k \ell}=-6 \notin\{-3,-2,1\}$ which contradicts to (6.63). Consequently, we know that Case (ii-b) occurs only under the condition: $\ell=3$.

Without loss of generality, we may assume that $\gamma_{12}<\gamma_{13}<\gamma_{23}$.

If $\gamma_{23}<0$, we obtain from $(6.64)$ that $\gamma_{12}+\gamma_{13}+\gamma_{23} \leq-\frac{7}{2}$ which contradicts (6.63). Hence, we must have $\gamma_{23}=1$ and $\gamma_{12}+\gamma_{13} \in\{-4,-3\}$. Thus, by (6.64), we know that $\left(\gamma_{12}, \gamma_{13}\right)$ is $(-3,-1),\left(-\frac{5}{2},-\frac{1}{2}\right)$ or $(-2,-1)$. In all cases, we have $\gamma_{23}-\gamma_{13}-\gamma_{12} \in\{4,5\}$, which contradicts to (6.63). Hence, Case (ii-b) is impossible.

In summary, we have $\ell=3$ and $\gamma_{12}=\gamma_{13}=1$ or $\gamma_{12}=\gamma_{13}=-1$.

If $\gamma_{12}=\gamma_{13}=-1$ holds, by considering $\gamma_{23}-\gamma_{13}-\gamma_{12}$, we obtain from (6.63) and (6.64) that $\gamma_{23}=1$. Hence, we get $\gamma_{23}-\gamma_{12}-\gamma_{13}=3$ which is impossible due to (6.63). Thus, we find $\gamma_{12}=\gamma_{13}=1$. So, by using $\gamma_{23}+\gamma_{13}+\gamma_{12} \in\{-3,-2,1\}$, we obtain from (6.63) and (6.64) that $\gamma_{23}=-1$. Consequently, we have $\left(\gamma_{12}, \gamma_{13}, \gamma_{23}\right)=(1,1,-1)$. Since $\ell=3$, it follows from (6.58) that $T^{2} \subset S^{5} \subset \mathbb{E}^{6}$. Thus, we may assume the immersion of $T^{2}$ in $\mathbb{E}^{6}$ is

$$
\begin{array}{r}
\mathbf{x}(s, t)=\left(\mu_{1} \sin \left(\bar{\alpha}_{1} s+\bar{p}_{1} t\right), \mu_{1} \cos \left(\bar{\alpha}_{1} s+\bar{p}_{1} t\right),\right. \\
\mu_{2} \sin \left(\bar{\alpha}_{2} s+\bar{p}_{2} t\right), \mu_{2} \cos \left(\bar{\alpha}_{2} s+\bar{p}_{2} t\right), \\
\left.\mu_{3} \sin \left(\bar{\alpha}_{3} s+\bar{p}_{3} t\right), \mu_{3} \cos \left(\bar{\alpha}_{3} s+\bar{p}_{3} t\right)\right) .
\end{array}
$$

We may assume $\bar{p}_{3}=0$ by applying a suitable rotation on the $(s, t)$-plane if necessary. From the discussions above, we know that $\bar{\alpha}_{1}, \bar{\alpha}_{2}, \bar{\alpha}_{3}, \bar{p}_{1}, \bar{p}_{2}$ satisfy

$$
\begin{aligned}
& \bar{a}_{1} \bar{a}_{2}+\bar{p}_{1} \bar{p}_{2}=\bar{a}_{1} \bar{a}_{3}=-\bar{a}_{2} \bar{a}_{3}=1, \\
& \bar{\alpha}_{1}^{2}+\bar{p}_{1}^{2}=\bar{\alpha}_{2}^{2}+\bar{p}_{2}^{2}=\bar{\alpha}_{3}^{2}=2 .
\end{aligned}
$$


After solving this system we obtain

$$
\sqrt{2}\left(\bar{\alpha}_{1}, \bar{\alpha}_{2}, \bar{\alpha}_{3}, \bar{p}_{1}, \bar{p}_{2}\right)= \pm(1,-1,2, \sqrt{3}, \sqrt{3}) .
$$

Hence, up to rigid motions, the immersion of $T^{2}$ in $S^{5} \subset \mathbb{E}^{6}$ takes the form:

$$
\begin{aligned}
& \mathbf{x}(s, t)=\left(\mu_{1} \cos \frac{1}{\sqrt{2}}(\sqrt{3} t+s), \mu_{1} \sin \frac{1}{\sqrt{2}}(\sqrt{3} t+s),\right. \\
& \left.\mu_{2} \cos \frac{1}{\sqrt{2}}(\sqrt{3} t-s), \mu_{2} \sin \frac{1}{\sqrt{2}}(\sqrt{3} t-s), \mu_{3} \cos \sqrt{2} s, \mu_{3} \sin \sqrt{2} s\right)
\end{aligned}
$$

for some positive numbers $\mu_{1}, \mu_{2}, \mu_{3}$. Finally, by applying (6.60), we obtain $\mu_{1}=\mu_{2}=\mu_{3}=\frac{1}{\sqrt{3}}$. Consequently, the flat minimal torus $T^{2}$ in $S^{5}$ is congruent to the equilateral minimal torus defined by (6.1).

\section{Some additional results on spherical Gauss map}

The next result provides some answers to the following question:

Question: When the spherical Gauss map of a spherical minimal surface is minimal?

Proposition 7.1. If $\mathbf{x}:\left(M^{2}, g\right) \rightarrow S^{m-1}$ is a non-totally geodesic minimal surface, then its spherical Gauss map $\hat{\nu}:\left(M^{2}, \hat{g}\right) \rightarrow S^{N-1}$ with $N=\left(\begin{array}{c}m \\ 3\end{array}\right)$ is a minimal surface of $S^{N-1}$ if and only if any one of the following three conditions holds:

(1) $M^{2}$ is a minimal surface of a totally geodesic 3-sphere $S^{3} \subset S^{m-1}$.

(2) $\mathrm{x}:\left(M^{2}, g\right) \rightarrow S^{m-1}$ has flat normal connection.

(3) $\hat{\nu}:\left(M^{2}, \hat{g}\right) \rightarrow S^{N-1}$ has parallel mean curvature vector.

Proof. Let $\mathbf{x}:\left(M^{2}, g\right) \rightarrow S^{m-1}$ be a non-totally geodesic minimal surface and let $\hat{\Delta}$ be the Laplacian operator of $\left(M^{2}, \hat{g}\right)$. Then the totally geodesic points are isolated. If $\hat{\nu}:\left(M^{2}, \hat{g}\right) \rightarrow S^{N-1}$ of $\mathrm{x}$ is minimal in $S^{N-1}$, then condition (3) holds trivially.

Now, let us choose $e_{3}, \ldots, e_{m-1}$ such that $h_{i j}^{r}=0$ for $r=5, \ldots, m-1$. Then we have (5.10). Since the induced metric $\hat{g}=(1-K) g$ via $\hat{\nu}$ is conformal to the original metric $g$ on $M^{2}$ according to (3.8), we obtain from (5.10) that

$$
\hat{\Delta} \tilde{\nu}=-2 \tilde{\nu}+\left(\frac{2 K^{D}}{1-K}\right) \times \wedge e_{3} \wedge e_{4} .
$$

Thus, the mean curvature vector $\tilde{H}$ of $\tilde{\nu}:\left(M^{2}, \hat{g}\right) \rightarrow S^{N-1}$ is

$$
\tilde{H}=\left(\frac{2 K^{D}}{1-K}\right) \times \wedge e_{3} \wedge e_{4}
$$

Hence, $\hat{\nu}: M^{2} \rightarrow S^{N-1}$ is minimal if and only if condition (2) holds.

Conditions (1) and (2) are equivalent, since minimal surfaces in $S^{m-1}$ with flat normal connection are minimal surfaces lying in a totally geodesic $S^{3} \subset$ $S^{m-1}$. 
Now, assume that condition (3) holds. Then we have

$$
\tilde{\nabla}_{X} \tilde{H}=\left\langle\tilde{\nabla}_{X} \tilde{H}, f_{1}\right\rangle f_{1}+\left\langle\tilde{\nabla}_{X} \tilde{H}, f_{2}\right\rangle f_{2},
$$

where $f_{1}, f_{2}$ are orthonormal vector fields of $(\tilde{\nu}(M), \hat{g})$. From (7.2) we have

$$
\begin{aligned}
\tilde{\nabla}_{X} \tilde{H}= & 2 X\left(\frac{K^{D}}{1-K}\right) \mathbf{x} \wedge e_{3} \wedge e_{4} \\
& +\frac{2 K^{D}}{1-K}\left\{X \wedge e_{3} \wedge e_{4}-\mathbf{x} \wedge A_{e_{r}} \mathbf{x} \wedge e_{4}\right. \\
& \left.-\mathbf{x} \wedge e_{3} \wedge A_{e_{4}} X+\mathbf{x} \wedge D_{X} e_{3} \wedge e_{4}+\mathbf{x} \wedge e_{3} \wedge D_{X} e_{4}\right\}
\end{aligned}
$$

for $X \in T M^{2}$, which shows that $f_{1}, f_{2}$ are linear combinations of

$$
\mathbf{x} \wedge e_{r} \wedge e_{2}, \quad \mathbf{x} \wedge e_{1} \wedge e_{r}, \quad r=3, \ldots, m-1 .
$$

Thus, (7.3) and (7.4) yield $K^{D}=0$, i.e., the normal connection of $\mathbf{x}$ is flat. Therefore, we obtain condition (1).

The next result provides a general property of the spherical Gauss map for spherical minimal surfaces.

Proposition 7.2. If $\left(M^{2}, g\right)$ is a minimal surface of $S^{m-1}$, then the mean curvature vector $\tilde{H}$ of $\hat{\nu}:(M, \hat{g}) \rightarrow S^{\left(\begin{array}{c}m \\ 3\end{array}\right)-1}$ satisfies $|\hat{H}| \leq 2$, with the equality holding if and only if the immersion is isotropic.

Proof. As we did in the proof of Theorem 4.1, let us choose $e_{3}, \ldots, e_{m-1}$ with $h_{i j}^{r}=0$ for $r=5, \ldots, m-1$. Let us also choose $e_{1}, e_{2}$ which diagonalize the shape operator $A_{e_{3}}$. Since we have $K=1-\frac{1}{2}\|\hat{h}\|^{2}$ from Gauss' equation, we find

$$
\begin{aligned}
& (1-K)^{2}-\left(K^{D}\right)^{2} \\
= & \left\{\left(h_{11}^{3}\right)^{2}+\left(h_{11}^{4}\right)^{2}+\left(h_{12}^{4}\right)^{2}\right\}^{2}-4\left(h_{11}^{3} h_{12}^{4}\right)^{2} \\
= & \left\{\left(h_{11}^{3}\right)^{2}-\left(h_{12}^{4}\right)^{2}\right\}^{2}+\left(h_{11}^{4}\right)^{4}+2\left(h_{11}^{4}\right)^{2}\left(\left(h_{11}^{3}\right)^{2}+\left(h_{12}^{4}\right)^{2}\right) .
\end{aligned}
$$

So, we obtain $|\tilde{H}| \leq 2$ by (7.1), with equality holding if and only if $\left|h_{11}^{3}\right|=\left|h_{12}^{4}\right|$ and $h_{11}^{4}=0$. The later conditions are equivalent to the isotropy of $M^{2}$ in $S^{m-1}$.

Finally, we give the following.

Proposition 7.3. If $\mathbf{x}:\left(M^{3}, g\right) \rightarrow S^{4}$ is minimal without totally geodesic points, then the scalar curvature of $\left(M^{3}, \hat{g}\right)$ equals to 6 .

Proof. We can identify $\tilde{\nu}$ as the unit normal vector field $e_{4}$ of $M^{3}$ in $S^{4}$. Let $h_{i j}=: h_{i j}^{4}$ and $\left(h^{i j}\right)$ the inverse matrix of $\left(h_{i j}\right)$. If we put $f_{i}=\sum_{j} h^{i j} e_{j}$, then we get $\left(d e_{4}\right)\left(f_{i}\right)=-e_{i}$. Thus, $f_{1}, f_{2}, f_{3}$ form an orthonormal basis on $\left(M^{3}, \hat{g}\right)$. 
Since $\left\langle d x, f_{i}\right\rangle=\sum_{j} h^{i j} \omega^{j}$, the matrix $\left(h^{i j}\right)$ is the matrix of the second fundamental form of $\hat{\nu}: M^{3} \rightarrow S^{4}$ with respect to $f_{1}, f_{2}, f_{3}$. Thus, from the equation of Gauss we know that the curvature tensor $\hat{R}$ of $\left(M^{3}, \hat{g}\right)$ satisfies

$$
\hat{R}_{j k \ell}^{i}=\delta_{i k} \delta_{j \ell}-\delta_{i \ell} \delta_{j k}+h^{i k} h^{j \ell}-h^{i \ell} h^{j k} .
$$

Hence, the scalar curvature $\hat{S}$ of $\left(M^{3}, \hat{g}\right)$ is

$$
\hat{S}=6+\left(\sum_{j} h^{j j}\right)^{2}-\sum_{i, j}\left(h^{i j}\right)^{2} .
$$

If $e_{1}, e_{2}, e_{3}$ diagonalize $\left(h_{i j}\right)$, then we find

$$
\hat{S}=6+\frac{2 \sum_{j} h_{j j}}{h_{11} h_{22} h_{33}}=6 .
$$

This proves the proposition.

\section{References}

[1] C. Baikoussis, Ruled submanifolds with finite type Gauss map, J. Geom. 49 (1994), no. 1-2, 42-45.

[2] C. Baikoussis and D. E. Blair, On the Gauss map of muled surfaces, Glasgow Math. J. 34 (1992), no. 3, 355-359.

[3] C. Baikoussis, B. Y. Chen and L. Verstraelen, Ruled surfaces and tubes with finite type Gauss map, Tokyo J. Math. 16 (1993), no. 2, 341-349.

[4] C. Baikoussis and L. Verstraelen, On the Gauss map of helicoidal surfaces, Rend. Sem. Mat. Messina Ser. II 16 (1993), 31-42.

[5] B. Y. Chen, Geometry of submanifolds, Pure and Applied Mathematics, No. 22, Mercer Dekker, New York, 1973.

[6] __ Total mean curvature and submanifolds of finite type, Series in Pure Mathematics, 1, World Scientific, New Jersey, 1984.

[7] _ A report on submanifolds of finite type, Soochow J. Math. 22 (1996), no. 2, $117-337$.

[8] _ Riemannian Submanifolds, in Handbook of Differential Geometry, vol. I, North Holland, (edited by F. Dillen and L. Verstraelen) 2000, pp. 187-418.

[9] B. Y. Chen and S. J. Li, Spherical hypersurfaces with 2-type Gauss map, Beiträge Algebra Geom. 39 (1998), no. 1, 169-179.

[10] B. Y. Chen, M. Choi and Y. H. Kim, Surfaces of revolution with pointwise 1-type Gauss map, J. Korean Math. Soc. 42 (2005), no. 3, 447-455.

[11] B. Y. Chen and P. Piccinni, Submanifolds with finite type Gauss map, Bull. Austral. Math. Soc. 35 (1987), no. 2, 161-186.

[12] F. Dillen, J. Pas and L. Verstraelen, On the Gauss map of surfaces of revolution, Bull. Inst. Math. Acad. Sinica 18 (1990), no. 3, 239-246.

[13] K. O. Jang and Y. H. Kim, 2-type surfaces with 1-type Gauss map, Commun. Korean Math. Soc. 12 (1997), no. 1, 79-86.

[14] K. Kenmotsu, On minimal immersions of $R^{2}$ into $S^{n}$, J. Math. Soc. Japan 28 (1976), no. 1, 182-191.

[15] _ On Veronese-Borivka spheres, Arch. Math. (Brno) 33 (1997), no. 1-2, 37-40.

[16] _ Minimal surfaces with constant curvature in 4-dimensional space forms, Proc. Amer. Math. Soc. 89 (1983), no. 1, 133-138.

[17] Y. H. Kim and D. W. Yoon, Ruled surfaces with pointwise 1-type Gauss map, J. Geom. Phys. 34 (2000), no. 3-4, 191-205. 
[18] H. B. Jr. Lawson, Complete minimal surfaces in $S^{3}$, Ann. of Math. (2) 92 (1970), 335-374.

[19] M. Obata, The Gauss map of immersions of Riemannian manifolds in space of constant curvature, J. Differential Geometry 2 (1968), 217-223.

[20] R. Osserman, Minimal surfaces, Gauss maps, total curvature, eigenvalues estimates and stability, in: The Chern Symposium, pp. 199-227, Berkeley, 1979.

[21] E. A. Ruh and J. Vilms, The tension field of the Gauss map, Trans. Amer. Math. Soc. 149 (1970), 569-573.

[22] N. Wallach, Extension of locally defined minimal immersions into spheres, Arch. Math. (Basel) 21 (1970), 210-213.

BANG-YEN CHEN

DePaRtment of Mathematics

Michigan State University

EAST LANSING, MI 48824-1027, U.S.A.

E-mail address: bychen@math.msu.edu

HueI-SHyong Lue

DePartment of Computer SCIENCES AND INFormation EngINEERING

YUANPEI INSTITUTE OF SCIENCE AND TEChNOLOGY

HSINCHU, TAIWAN

E-mail address: hslue@math.nthu.edu.tw 